\title{
Boosting Biomass Quantity and Quality by Improved Mixotrophic Culture of the Diatom Phaeodactylum tricornutum
}

\begin{abstract}
Valeria Villanova ${ }^{1,2,3 * \dagger}$, Dipali Singh ${ }^{4,5 * t}$, Julien Pagliardini ${ }^{3}$, David Fell ${ }^{5}$, Adeline Le Monnier ${ }^{3}$, Giovanni Finazzi ${ }^{2}$ and Mark Poolman ${ }^{5}$

${ }^{1}$ Department of Biological and Environmental Sciences, University of Gothenburg, Gothenburg, Sweden, ${ }^{2}$ Laboratoire de Physiologie Cellulaire et Végétale, Université Grenoble Alpes (UGA), Centre National de la Recherche Scientifique (CNRS), Commissariat á l'Énergie Atomique et aux Énergies Alternatives (CEA), Institut National de Recherche pour l'Agriculture, l'Alimentation et l'Environnement, Interdisciplinary Research Institute of Grenoble, CEA Grenoble, Grenoble, France, ${ }^{3}$ Fermentalg SA, Libourne, France, ${ }^{4}$ Microbes in the Food Chain, Quadram Institute Biosciences, Norwich Research Park, Norwich, United Kingdom, ${ }^{5}$ Cell System Modelling Group, Oxford Brookes University, Oxford, United Kingdom
\end{abstract}

OPEN ACCESS

Edited by:

Richard Dorrell,

École Normale Supérieure, France

Reviewed by:

Pierre Cardol,

University of Liége, Belgium

Hanhua Hu,

Chinese Academy of Sciences, China

*Correspondence:

Valeria Villanova

valeria.villanova@bioenv.gu.se

Dipali Singh

dipali.singh@quadram.ac.uk

tThese authors have contributed equally to this work and share first authorship

Specialty section:

This article was submitted to Marine and Freshwater Plants,

a section of the journal

Frontiers in Plant Science

Received: 15 December 2020

Accepted: 22 February 2021

Published: 09 April 2021

Citation:

Villanova V, Singh D, Pagliardini J,

Fell D, Le Monnier A, Finazzi $G$ and

Poolman M (2021) Boosting Biomass

Quantity and Quality by Improved

Mixotrophic Culture of the Diatom

Phaeodactylum tricornutum.

Front. Plant Sci. 12:642199.

doi: 10.3389/fpls.2021.642199
Diatoms are photoautotrophic unicellular algae and are among the most abundant, adaptable, and diverse marine phytoplankton. They are extremely interesting not only for their ecological role but also as potential feedstocks for sustainable biofuels and high-value commodities such as omega fatty acids, because of their capacity to accumulate lipids. However, the cultivation of microalgae on an industrial scale requires higher cell densities and lipid accumulation than those found in nature to make the process economically viable. One of the known ways to induce lipid accumulation in Phaeodactylum tricornutum is nitrogen deprivation, which comes at the expense of growth inhibition and lower cell density. Thus, alternative ways need to be explored to enhance the lipid production as well as biomass density to make them sustainable at industrial scale. In this study, we have used experimental and metabolic modeling approaches to optimize the media composition, in terms of elemental composition, organic and inorganic carbon sources, and light intensity, that boost both biomass quality and quantity of $P$. tricornutum. Eventually, the optimized conditions were scaled-up to $2 \mathrm{~L}$ photobioreactors, where a better system control (temperature, $\mathrm{pH}$, light, aeration/mixing) allowed a further improvement of the biomass capacity of $P$. tricornutum to $12 \mathrm{~g} / \mathrm{L}$.

Keywords: genome-scale metabolic model, linear programming, metabolism, mixotrophic growth, diatom, $P$. tricornutum, biomass productivity

\section{INTRODUCTION}

Diatoms are photosynthetic unicellular microalgae that dominate the oceans. Their ability to synthesize lipid as a storage compound makes them a potential source of biofuel and high-value commodities such as omega fatty acids (Hildebrand et al., 2012; d'Ippolito et al., 2015; Wang and Seibert, 2017; Yi et al., 2017; Pudney et al., 2019). They have notably different evolutionary history from that of other photosynthetic eukaryotes such as plants and green algae, and are thought to have arisen from a complex endosymbiotic event, which is ascertained, though donor is not clearly identified (Wilhelm et al., 2006; Armbrust, 2009; Moustafa et al., 2009). Consequently, diatoms have a number of unique biochemical features distinguishing them other photosynthetic eukaryotes. 
Unlike other photosynthetic eukaryotes, enzymes associated with the Calvin cycle, in diatoms, are not solely located in the chloroplast; possibly the Calvin cycle enzyme sedoheptulosebisphosphatase (SBPase) is instead located in the cytosol (Kroth et al., 2008), suggesting that either other chloroplast enzymes compensate for the absence of SBPase, or that the Calvin cycle is distributed between the chloroplast and cytosol. Similarly, enzymes of the oxidative limb of the oxidative pentose phosphate pathway (OPPP) are located in the cytosol, not the plastid. In other photosynthetic eukaryotes, glycolysis follows the wellknown Embden-Meyerhof-Parnass (EMP) scheme and is located in the cytosol. The situation in Phaeodactylum tricornutum is somewhat different; the cytosolic pathway lacks the enzyme enolase, with the result that the cytosolic pathway is truncated at phosphoglycerate (PGA). However, enolase and pyruvate kinase are found in both the chloroplast and the mitochondrion leading to the possibility of glycolysis being distributed between two or even three subcellular compartments (Liaud et al., 2000; Kroth et al., 2008; Smith et al., 2012). The Entner-Doudoroff (ED) pathway and phosphoketolase pathways, commonly found in prokaryotes, have been reported to be present in P. tricornutum (Fabris et al., 2012), though the contribution of these pathways in $P$. tricornutum is not well-known.

Diatoms can produce biomass via different modes of growth: phototrophic and mixotrophic. The former mode simply converts the sunlight energy into reduced carbon via photosynthesis. This can be a cheap way to produce biomass because only sunlight is used as an energy source, but it is often limited by the difficulties in optimizing light penetration, gas diffusion, and temperature control in photobioreactors and race ponds (Chisti, 2007, 2008). While some microalgae can grow heterotrophically via sugar fermentation in the dark, this is not possible in Phaeodactylum, which can use sugars in the dark only upon metabolic engineering (Zaslavskaia et al., 2001). On the other hand, $P$. tricornutum can grow mixotrophically, i.e., using simultaneously light and reduced carbon (Garcì et al., 2004; Garcia et al., 2005, 2006, 2013; Liu et al., 2009a). This mode of cultivation represents an interesting alternative to phototrophic growth, because mixotrophically grown algae have, in principle, a lower requirement for optimum light penetration, and can make use of cheap and easily available carbon sources, like glycerol, leading to a high biomass productivity (Garcì et al., 2004; Garcìa et al., 2005, 2006, 2013).

Nitrogen and phosphorus (after carbon) are often the most important macronutrients required for the growth of microalgae (Wijffels and Barbosa, 2010) and the deprivation of these elements has been reported to trigger TAG accumulation in Phaeodactylum (Abida et al., 2015). However, deprivation of these elements also restricts growth, and thus biomass quantity. Micronutrients are essential for algal growth because of their role as cofactors of key enzymes (Morel et al., 2003; Merchant and Helmann, 2012; Blaby-Haas and Merchant, 2017). For instance, iron is a redox active metal present in several metalloproteins involved in photosynthesis, respiration, and nitrogen assimilation and hence is very important in phototrophs (Moore et al., 2013). Manganese also plays a very important role (Sunda and Huntsman, 1996). Indeed, the manganese is involved in such vital processes of phototrophic organisms as the oxidation of water done by the PSII complex, and the conversion of superoxide radical into molecular oxygen $\left(\mathrm{O}_{2}\right)$ and hydrogen peroxide (Blaby-Haas and Merchant, 2017; Saavedra et al., 2018). Zinc is also essential, as shown by the examples of the green alga Chlamydomonas reinhardtii, where it impacts $\mathrm{CO}_{2}$ assimilation and $\mathrm{Cu}$ homeostasis (Malasarn et al., 2013), and of the diatom T. weissflogii, where it seems to be associated with carbonic anhydrase (CA) regulating the rate of carbon uptake and fixation (Morel et al., 1994). Copper has also an effect on diatoms' growth, possibly because this element is involved in the regulation of Fe uptake (Annett et al., 2008) and, in some cases, in the substitution of $\mathrm{Fe}$-containing enzymes by $\mathrm{Cu}$-containing enzymes (e.g., plastocyanin in T. oceanica) (Peers and Price, 2006). However, too elevated concentrations of these elements may result in oxidative damage or in decrease of growth rate of cells (Choudhary et al., 2007).

The f/ 2 medium by Guillard (1975) (half the concentration of the original $\mathrm{f}$ medium designed by Guillard and Ryther, 1962) is one of the earliest designed and a widely used enriched seawater medium for growing coastal marine algae, especially diatoms. It is composed of filtered natural seawater, trace elements, and vitamins. However, the use of natural seawater can result in seasonal variability and difference in quality, which led to design of artificial seawater medium to provide better quality control. Enriched seawater artificial seawater (ESAW) (Harrison et al., 1980) is another extensively used medium for microalgae physiological study. It composition is constant and its use is not detrimental for the algae. Some microalgae can reach a higher biomass yield in ESAW than in the $\mathrm{f} / 2$ medium. The ESAW medium was later modified by Berges et al. (2001), for salts and metals, leading to improved marine algal growth. For these reasons, we decided to use the modified ESAW as the starting medium in this study.

Light is another important parameter to consider for the optimization of phototrophic and mixotrophic algal cultivation (Falkowski and Owens, 1980; Rashid et al., 2015). Increasing the light intensity usually tends to increment the algal growth rate up to the light saturation level. However, when light becomes oversaturating, it can lead to the formation of harmful products (i.e., ROS) and, hence, decrease algal biomass productivity (Richmond, 2000). To avoid this phenomenon of photoinhibition, light intensity has to be optimized in relation to cell density, so that the culture does not become light limited or photodamaged (Eriksen, 2008; Zhu, 2015; Sivakaminathan et al., 2018). The optimization of medium composition for microand macronutrients, carbons source and light intensity is a crucial step for enhancing the biomass productivity and lipid concentration of the selected microalgae.

The genome-encoded metabolic potential, as a genome-scale metabolic model (GSM), has been previously exploited to define optimal conditions, design growth media, and predict metabolic fluxes under different genetic and environmental conditions (Kim et al., 2017; Pan and Reed, 2018; Gu et al., 2019; Ong et al., 2020; Tejera et al., 2020). Its analysis enables an investigation of metabolic behavior of the whole system and has been utilized to inform experimental design and to provide a rationale for 
experimental observations (McCloskey et al., 2013; Villanova et al., 2017; Mishra et al., 2018; van der Ark et al., 2018).

In this work, we have used a genome-scale metabolic model and an experimental approach to test the role of inorganic and organic carbon (glycerol) sources in P. tricornutum biomass productivity and optimized the elemental composition of macroand micronutrients in the ESAW medium (Berges et al., 2001) in order to enhance both biomass quantity and quality. The optimized medium composition was used to scale-up $P$. tricornutum culture to a $2-\mathrm{L}$ photobioreactor where light intensity was further optimized for cell density enhancement. Using our enhanced optimized media, biomass and fatty acid production in P. tricornutum increased by a factor of about 9 and 45 , respectively.

\section{MATERIALS AND METHODS}

\subsection{Strains, Growth Media, and Culture Conditions}

Axenic cultures of P. tricornutum (Pt1, CCAP 1055/3, Martino et al., 2007) were grown in a $250 \mathrm{~mL}$ Erlenmeyer flask in an artificial seawater ESAW (Berges et al., 2001) supplemented with extra $\mathrm{NaNO}_{3}$ and $\mathrm{NaH}_{2} \mathrm{PO}_{4}$ to reach a final concentration of 0.47 $\mathrm{g} / \mathrm{L} \mathrm{N}$ and $0.03 \mathrm{~g} / \mathrm{L} \mathrm{P}$ (this medium will be referred as E10 from now on). Cells were grown in a chamber at $20^{\circ} \mathrm{C}, 40 \mu \mathrm{E} \mathrm{m}^{-2} \mathrm{~s}^{-1}$ irradiance with a led light for 12-h light/12-h dark photoperiod and shaking at $100 \mathrm{rpm}$. For mixotrophic growth experiments, filter sterilized glycerol was added at a final concentration of 4.6 g/L (E10+GLY).

To monitor algal growth, samples were taken daily (in the end of the light period) and growth was estimated by (i) calculating dry cell weight (DCW) and (ii) measuring the optical density at $750 \mathrm{~nm}$ using a double beam UV/visible spectrophotometer from Thermo Fisher Scientific. In the latter case, cell concentration was evaluated from a calibration curve obtained by the correlation of the absorbance at $750 \mathrm{~nm}$ and dry weight (Supplementary File 1). The growth profiles in the different media were compared as a function of $\operatorname{Ln}(\mathrm{C} / \mathrm{C} 0)$, where $\mathrm{C}$ is the biomass concentration at a certain time, $\mathrm{t}$, and $\mathrm{C} 0$ is the initial biomass concentration.

\subsubsection{Dry Cell Weight Protocol}

A millipore membrane filter (diameter $47 \mathrm{~mm}, \mu=0.45 \mu \mathrm{m}$ ) placed in an aluminum cup $(\varnothing \mathrm{xh}=70 \times 6 \mathrm{~mm})$ was dried $24 \mathrm{~h}$ in an oven and weighed. Note that $2-10 \mathrm{~mL}$ of culture was filtered through a dried filter. The filter was rinsed with sea water $(2 \mathrm{x}$ filtered volume). Finally, the filter + biomass was dried for $24 \mathrm{~h}$ and weighed again. The DCW was calculated as: [weight of (filter + aluminum cup + biomass) $]-$ [weight of (filter + aluminum cup)]/L of filtered culture and expressed as $\mathrm{g} / \mathrm{L}$.

\subsubsection{Scale-Up in 2-L Photobioreactor}

The optimized conditions were carried out in duplicate in 2L photobioreactors (Applikon Schiedam, The Netherlands). All cultures were sparged continuously with air at flow rate of 0.5 $\mathrm{L} / \mathrm{min}$. The $\mathrm{pH}$ was controlled at 8 by automatic on-demand injection of $0.25 \mathrm{~N}$ of $\mathrm{HNO}_{3}$. Temperature was controlled at $20^{\circ} \mathrm{C}$ by circulating water. Light was supplied continuously with external light panels ranging from 70 to $300 \mu \mathrm{E} \mathrm{m}^{-2} \mathrm{~s}^{-1}$. The cultures were ensured to be axenic by checking the cellular morphology using 100x microscopy every day.

\subsection{Photosynthesis and Respiration Measurements}

\subsubsection{Chlorophyll Fluorescence}

All the photosynthetic parameters were determined using a Speedzen MX fluorescence imaging setup (JBeamBio, France) as described in Vandystadt et al. (2009). For these experiments, the first version of the Speed Zen (which has no number) was used. In this setup, actinic light and saturating pulses are provided by green LEDs peaking at $520 \mathrm{~nm}$, while measuring pulses (duration $250 \mathrm{~ms}$ ) are provided by blue LEDs peaking at $470 \mathrm{~nm}$. The detection time after every detecting pulse was $15 \mu \mathrm{s}$. No binning was applied.

For each sample, $3 \times 200 \mu \mathrm{L}$ of algal culture were transferred in a 96-well plate. Maximum quantum yield of PSII (Fv/Fm $=(\mathrm{Fm}$ - F0)/Fm) was determined after 15 min of dark incubation, where Fm and F0 are the maximum and minimum fluorescence of dark-adapted cells, respectively. Nonphotochemical quenching (NPQ) was calculated as (Fm-Fm')/Fm', where Fm' and Fm are maximum fluorescence of light-adapted and dark-adapted cells, respectively. NPQ was measured at $400 \mu \mathrm{E}$. This light intensity was chosen after preliminary experiments on light dependency of NPQ that showed $400 \mu \mathrm{E}$ was enough to reach the maximum value, without inducing photodamage.

\subsubsection{Respiration Rates}

Respiration rates were measured as $\mathrm{O}_{2}$ exchange rates using a Clark-type oxygen electrode at $19^{\circ} \mathrm{C}$ (Hansatech Instruments). The cells from the exponential phase of flask experiment were collected, at day 4 , and the concentration adjusted to $3^{\star} 10^{7}$ cells/mL with respective medium (i.e., E10: ESAW medium with 10 times extra $\mathrm{N}$ and $\mathrm{P}$ as described in section 2.1, E10+GLY: E10 supplemented with glycerol, EE: ESAW enriched medium developed in this study as described in section 3.2.1, EE+GLY: EE supplemented with glycerol, EE+BIC: EE supplemented with bicarbonate, $\mathrm{EE}+\mathrm{BIC}+\mathrm{GLY}$ : EE supplemented with bicarbonate and glycerol). The $\mathrm{O}_{2}$ in the medium was calculated in both light (i.e., photosynthesis) and dark (i.e., respiration). The light intensity used for the determination of $\mathrm{O}_{2}$ in the photosynthesis was $200 \mu \mathrm{E} \mathrm{m}^{-2} \mathrm{~s}^{-1}$, which was enough to reach the maximum value as per our preliminary experiments.

\subsection{Metabolite Analysis}

\subsubsection{Nitrogen and Phosphate Concentration}

Nitrogen and phosphate concentration in supernatant were determining using a Merk RQflex reflectometer (E. Merck, 64271 Domsstadt, Germany) with test strips (Reflectoquant nitrate and phosphate).

\subsubsection{Glycerol Concentration}

The glycerol concentration of $2 \mathrm{~mL}$ of filtered supernatant was measured with a Shimadzu HPLC with a Hi-plex $\mathrm{H}+(7.7 \mathrm{X}$ $300 \mathrm{~mm}$ ) Agilent column. The analysis was performed using the 
mobile phase $5 \mathrm{mM} \mathrm{H}_{2} \mathrm{SO}_{4}$. The detection wavelength was set at $210 \mathrm{~nm}$ using a RI RID-10A Detector with a flow rate of 0.6 $\mathrm{mL} / \mathrm{min}$ and a temperature of $60^{\circ} \mathrm{C}$. The peak quantification was performed by comparison with a range of 6 solution standards.

\subsubsection{Total Lipids Extraction}

Total lipids were extracted according to Folch et al. (1957). About $20 \mathrm{mg}$ of dried cells were homogenized with $1 \mathrm{~mL}$ of chloroform/methanol 2:1. The cells were then lysed using a TissueLyser II (Qiagen) with an agitation of $1 \mathrm{~min}$ and a frequency of $300 \mathrm{~s}^{-1}$. The lysate was washed with $200 \mu \mathrm{L}$ of $\mathrm{NaCl} 0.9 \%$ and vortexed for some seconds in order to form the emulsion. The solution was centrifuged $5 \mathrm{~min}$ at 13,000 rpm to separate the two phase and the lower phase placed in fresh pre-weighed glass tubes. The upper phase was washed with chloroform; lysis and centrifugation steps were repeated in order to recover more lipids. The wash with chloroform was repeated at least twice. The lower phases (containing lipids) collected in glass tubes were evaporated under a nitrogen stream at $65^{\circ} \mathrm{C}$. The glass tubes were weighed to determine the percentage of lipids extracted per dry cells.

\subsubsection{Fatty Acid Determination}

For the fatty acid isolation, $1 \mathrm{~mL}$ of chloroform was added to 2 $\mathrm{mg}$ of freeze-dried biomass in $7-\mathrm{mL}$ glass tubes. The tubes were heated in a shaking water bath at $80^{\circ} \mathrm{C}$ for $90 \mathrm{~min}$. Note that $1.5 \mathrm{~mL}$ of ultra-pure water and $2 \mathrm{~mL}$ of heptane were added to the tubes, once cooled to the room temperature, and mixed by vortexing. The tubes were then centrifuged for $5 \mathrm{~min}$ at $3,000 \mathrm{rpm}$ to separate the phase. The upper phase containing heptane and fatty acid methyl ester (FAMEs) was recovered in a labeled screw vial. Note that $10 \mathrm{mg}$ of C23:0-Me was added to the samples and used as internal standard. Finally, the samples were injected in a gas chromatography coupled with a flame ionization detector (GC-FID) from Shimadzu and a $30 \mathrm{~m} \times 0.25 \mathrm{~mm} \times 0.25 \mu \mathrm{m}$ DB-23 capillary column from Agilent. FAMEs were identified by GC-FID and compared with different standards (Supelco 37 component FAME Mix and PUFA No. 3, from Menhaden). The FAMEs were quantified through the comparison against the C23:0 internal standard.

\subsubsection{Pigment Determination}

The pigment extraction was carried out under hood in the dark. Two to three milligram of freeze-dried biomass was dissolved in $1 \mathrm{~mL}$ of $95 \%$ ethanol/acetonitrile (60/40) in $2 \mathrm{~mL}$ "Safe Lock" eppendorf and stainless-steel beads were added. The tubes were ground in a TissueLyser II QUIAGEN for $1 \mathrm{~min}$ at $30 \mathrm{~Hz} \mathrm{~s}^{-1}$. The tubes were then placed in an ultrasonic water bath for 10 min and centrifuged at 13,000 rpm for $5 \mathrm{~min}$. If the pellet was white, $900 \mu \mathrm{L}$ of supernatant was placed in a labeled amber vial and $180 \mu \mathrm{L}$ of water was added. Otherwise, the supernatant was collected in a separate tube, new solvent was added, and ultrasonication was repeated until the pellet was colorless. The pigment determination was carried out with a HPLC DIONEX Ultimate 3000 and a detector DAD 3000. The reverse phase was the column C8 Agilent Elipse XDB $3.5 \mu \mathrm{m}(4.6 \times 150 \mathrm{~mm})$ with pre-column C8 XDB Agilent (4.6 x $12.4 \mathrm{~mm})$. Solvent A consisted of ethanol 50\%/28 mM tetra-butyl ammonium acetate at $\mathrm{pH} 6.5(70 / 30)$ and solvent $\mathrm{B}$ of ethanol/ACN (60/40). The elution gradient was: 0 min: $95 \% \mathrm{~A}$ and $5 \% \mathrm{~B} ; 3$ min: $60 \% \mathrm{~A}$ and $40 \% \mathrm{~B} ; 7$ min: $60 \% \mathrm{~A}$ and $40 \% \mathrm{~B} ; 21 \mathrm{~min}: 20 \% \mathrm{~A}$ and $80 \%$ B; 26 min: $20 \% \mathrm{~A}$ and $80 \%$ B; 27 min: $5 \% \mathrm{~A}$ and $95 \% \mathrm{~B}$; 32 min: $5 \% \mathrm{~A}$ and $95 \% \mathrm{~B} ; 37 \mathrm{~min}: 95 \% \mathrm{~A}$ and $5 \% \mathrm{~B} ; 40 \mathrm{~min}$ : $95 \% \mathrm{~A}$ and $5 \% \mathrm{~B}$. The flow rate was kept at $1 \mathrm{~mL} / \mathrm{min}$ and the column was set at $60^{\circ} \mathrm{C}$. Pigments were detected by diode-array spectroscopy (wavelength range: $280-800 \mathrm{~nm}$ ) with comparison of the standards. For the identification and the quantification of the pigments the following standards were used; $\beta$-carotene (Fluka, ref.: 1448298), Fucoxanthin (Sigma-Aldrich, ref.: F6932$10 \mathrm{MG}$ ) and a mix of pigments derived from the extract of a strains present in FERMENTALG culture collection.

\subsubsection{Carbohydrate Determination}

For the carbohydrate determination, $250 \mu \mathrm{L}$ of $80 \%$ sulfuric acid $\left(\mathrm{H}_{2} \mathrm{SO}_{4}\right)$ was added to $2 \mathrm{mg}$ of freeze-dried biomass in 7 $\mathrm{mL}$ screw cap glass tubes to hydrolyze samples. The tubes were centrifuged for $1 \mathrm{~min}$ at $3000 \mathrm{rpm}$ and then heated in a dry bath at $110^{\circ} \mathrm{C}$ for about $1 \mathrm{~min}$. The samples were placed for 15 min in an ultrasonic bath. Blank, standards, and samples were prepared in new glass tubes for the spectrophotometer analysis as follows: Blank: $15 \mu \mathrm{L}$ of ultrapure water $+720 \mu \mathrm{L}$ of $0.625 \%$ phenol $+1.5 \mathrm{~mL}$ of $80 \% \mathrm{H}_{2} \mathrm{SO}_{4}$, standards: $15 \mu \mathrm{L}$ of standards solution $+720 \mu \mathrm{L}$ of $0.625 \%$ phenol $+1.5 \mathrm{~mL}$ of $80 \% \mathrm{H}_{2} \mathrm{SO}_{4}$, sample: $15 \mu \mathrm{L}$ of sample $+720 \mu \mathrm{L}$ of $0.625 \%$ phenol $+1.5 \mathrm{~mL}$ of $80 \% \mathrm{H}_{2} \mathrm{SO}_{4}$. The tubes were then vortexed and placed in a dry bath at $110^{\circ} \mathrm{C}$ for $5 \mathrm{~min}$ and finally the tubes were placed in an ice-water bath for $1 \mathrm{~min}$ to stop the reaction. Note that $150 \mu \mathrm{L}$ of sample was then placed in a 96-well plate and put in a plate reader spectrophotometer to read the absorbance at $492 \mathrm{~nm}$. Biomass concentration in the hydrolysis tube: $[\mathrm{B}]=(\mathrm{mg}$ of dried biomass x 1000)/volume of $\mathrm{H}_{2} \mathrm{SO}_{4} 80 \%(250 \mu \mathrm{L})$. Quantity of biomass in the staining tube: $\mathrm{QB}=[\mathrm{B}](\mu \mathrm{g} / \mu \mathrm{L}) \mathrm{x}$ the hydrolyzate collected $(15 \mu \mathrm{L})$. Quantity of total carbohydrates in the tube: QC $=$ OD 492/coefficient of calibration obtained from the standard. Concentration of carbohydrates in biomass: [carbohydrates] $=$ $(\mathrm{QC} / \mathrm{QB}) \mathrm{x} 1000$. The standards used to generate the calibration curve were serial dilution of a glucose stock solution (from 4 to $0.07 \mathrm{~g}$ ).

\subsection{Statistical Analyses}

Growth profile $\operatorname{Ln}(\mathrm{C} / \mathrm{C} 0)$ data were fitted by linear regression between day 1 and 6 corresponding to the exponential phase in each medium. The slopes of the obtained regression curves, corresponding to the growth rate, were then compared by $t$ test analysis using GraphPad 9.01. The nutrient consumption was compared in mixotrophy and phototrophy growth in the different medium using two-way analysis of variance (ANOVA). For all the others analysis, i.e., biomass and photosynthesis, mixotrophy, and phototrophy conditions were compared using one-way ANOVA. $P$-values were used to quantify the variability between control (phototrophy) and treatment (mixotrophy) under different cultivation conditions. Data were considered significant for $p$-values $<0.1$. 


\subsection{Mathematical Modeling}

\subsubsection{Genome-Scale Metabolic Model}

A genome-scale metabolic model (GSM) of P. tricornutum developed in Singh et al. (2015) and Villanova et al. (2017), starting from the model of Hunt et al. (2014), was used in this study. The model consists of 450 reactions, 146 transporters (including external, inter-compartmental and biomass transporters), and 525 internal metabolites, and comprises cytosolic, plastidial, mitochondrial, and peroxisomal compartments. For this study, reactions were also balanced for proton and $\mathrm{O}_{2}$, in addition to all other atoms such as carbon, nitrogen, phosphorus, sulfur, and magnesium. It can use phosphate, sulfate, ammonium, nitrate, magnesium, and inorganic and/or organic carbon as input material for biomass production. It has been validated with respect to the laws of energy and mass conservation, and is able to produce all major biomass components (carbohydrate, lipid, amino acids, nucleotides, etc.) in phototrophic and mixotrophic conditions in experimentally observed proportions.

\subsubsection{Model Analysis: Linear Programming Formulation}

Model analysis was undertaken using the linear programming (LP) approach based on the law of mass conservation (Fell and Small, 1986; Varma and Palsson, 1994). The minimization of total flux in the network, as a proxy for economy of investment in enzymatic machinery (Holzhütter, 2006; Poolman et al., 2009), was used as the objective function along with the steadystate assumption and additional constraints, as defined in the following equation:

$$
\begin{array}{ll}
\text { minimize } & :|\mathbf{v}| \\
\text { subject to } & \left\{\begin{array}{l}
\mathbf{N v}=\mathbf{0} \\
\mathbf{v}_{v}=v \\
\mathbf{v}_{t x T A G}=T_{A G} \\
\mathbf{v}_{t x H C O 3}=H_{C O} \\
\mathbf{v}_{t x G l y c e r o l}=\text { Glycerol }
\end{array}\right.
\end{array}
$$

where $\mathbf{v}$ is the vector of all reaction fluxes and $\mathbf{N}$ is the stoichiometry matrix; the objective is to minimize the sum of all (absolute) flux values (including transporters), subject to the constraints: $\mathbf{N v}=\mathbf{0}$ (the steady-state assumption), $v_{v}=v$ which defines the photon flux (light) into the system, $v_{t x T A G}=$ TAG defines demand for TAG production, $v_{t x \mathrm{HCO}_{3}}=\mathrm{HCO}_{3}$ and $v_{\text {txGlycerol }}=$ Glycerol define constraints on $\mathrm{HCO}_{3}$ and glycerol transporters. Analysis under phototrophic condition has light as the only source of energy and $\mathrm{CO}_{2}$, and $\mathrm{HCO}_{3}$ where stated, as inorganic carbon source and no source of organic carbon (i.e., $v_{t x G l y c e r o l}=0$ ). Mixotrophic condition in this study refers to conditions with glycerol as organic carbon source. All computation was achieved using the ScrumPy metabolic modelling package (Poolman, 2006).
TABLE 1 | Objective value and number of reactions in linear programming solution for each condition.

\begin{tabular}{lcccc}
\hline & +BIC+GLY & -BIC+GLY & +BIC-GLY & -BIC-GLY \\
\hline No. of reactions & 69 & 103 & 67 & 95 \\
Objective value & $1.26 \times 10^{3}$ & $1.63 \times 10^{3}$ & $2.12 \times 10^{3}$ & $2.93 \times 10^{3}$ \\
\hline
\end{tabular}

TABLE 2 | Net production and consumption of substrates (net stoichiometry of linear programming solution) for each condition.

\begin{tabular}{lcccc}
\hline & +BIC+GLY & -BIC+GLY & +BIC-GLY & -BIC-GLY \\
\hline X_WATER & 26.857 & 48.676 & -32.0 & -47.99 \\
X_CARBON-DIOXIDE & 9.142 & 21.507 & -35.0 & -50.999 \\
X_HCO3 & -16.0 & 0.0 & -16.0 & 0.0 \\
X_GLYCEROL & -14.714 & -24.169 & 0.0 & 0.0 \\
X_TAG & 1 & 1 & 1 & 1 \\
x_Photon & -360.333 & -232.676 & -695.667 & -924.857 \\
X_OXYGEN-MOLECULE & 21 & -12.091 & 72.5 & 72.5 \\
x_PROTON & -15.999 & 0.0 & -16.0 & 0.0 \\
\hline
\end{tabular}

Here, negative value denotes consumption while positive value denotes production of substrates.

\section{RESULTS}

\subsection{Mathematical Modeling}

\subsubsection{Model Analysis in Phototrophic and}

\section{Mixotrophic Condition and the Effect of Bicarbonate}

The LP in Equation (1) was solved for 1 unit of TAG demand $\left(v_{t x T A G}=1.0\right)$ under four conditions: $(\mathrm{i})+\mathrm{BIC}+\mathrm{GLY}$ : mixotrophic condition in the presence of $\mathrm{HCO}_{3}$ (i.e., no constraint is set on glycerol and $\mathrm{HCO}_{3}$ transporters), (ii) BIC+GLY: mixotrophic condition in the absence of $\mathrm{HCO}_{3}$ ( $v_{t_{x} \mathrm{HCO}_{3}}=0$ while no constraint is set on glycerol transporters), (iii) +BIC-GLY: phototrophic condition in the presence of $\mathrm{HCO}_{3}$ (i.e., $v_{\text {txGlycerol }}=0$ while no constraint is set on $\mathrm{HCO}_{3}$ transporters) and, (iv) -BIC-GLY: phototrophic condition in the absence of $\mathrm{HCO}_{3}\left(v_{t x H C O}=v_{t x G l y c e r o l}=0\right)$.

The model is able to produce TAG under all four conditions; however, the objective value along with net consumption and production of substrates vary under these conditions and is summarized in Tables 1, 2, respectively. Availability of glycerol and/or $\mathrm{HCO}_{3}$ reduces the objective value and the requirement of light as energy source.

Glycerol, when available, is the preferred carbon source for lipid production. It enters metabolism through conversion to (i) glycerol phosphate, by glycerol kinase, which provides the immediate glycerol backbone for TAG synthesis, and (ii) DHAP, by glycerol-3-phosphate dehydrogenase, which enters the central carbon metabolism through glycolysis and cytosolic PPP. On the contrary, under phototrophic condition (in the absence of glycerol), carbon demand is met through inorganic carbon fixation through the Calvin cycle for which the enzymatic cost (objective value) and the requirement for light energy are higher compared to mixotrophic conditions, as shown in Tables 1, 2 , respectively. 


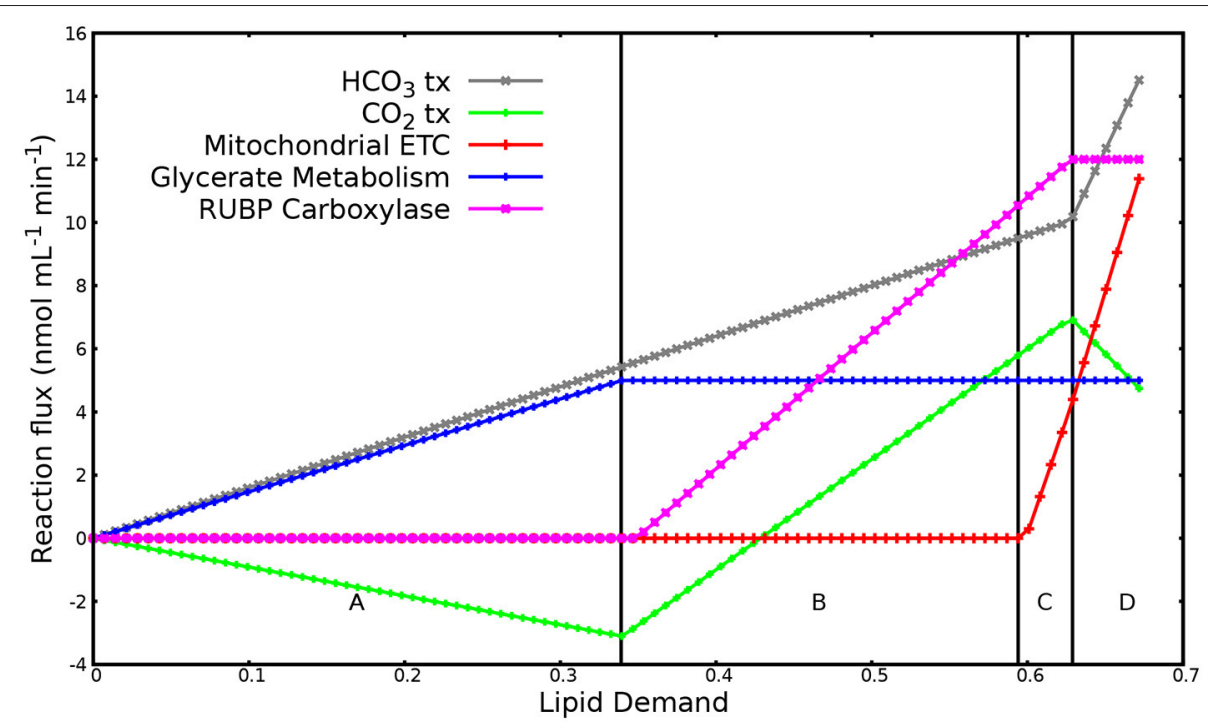

FIGURE 1 | Reaction responses to increase in lipid demand. The plot has been divided into four major regions labeled A to D based on the flux patterns. Region A: reaction response when flux in glycerol uptake, photon flux, and rubisco reaction is not saturated. Region B: reaction response when flux in glycerol transporter saturates. Region C: reaction response when photon flux saturates. Region D: reaction response when flux in Rubisco reaction saturates. Negative flux denotes export of substrate out of the system while positive flux denotes import of substrate into the system.

Bicarbonate, when available, is utilized by $\mathrm{PEP} /$ pyruvate carboxylase for anaplerotic production of C4 moieties from C3 moieties. The C3 skeleton (pyruvate and/or PEP) is produced through glycolysis under mixotrophic conditions and the Calvin cycle followed by lower glycolysis under phototrophic conditions. Anaplerotic fixation of $\mathrm{HCO}_{3}$ contributes one-third of carbon demand under both conditions (Table 2). Interestingly, in the absence of $\mathrm{HCO}_{3}$, photorespiration is active under both conditions. Glycolate produced through photorespiration is converted to glyoxylate, by glycolate dehydrogenase, that then enters the glyoxylate shunt, along with acetyl-CoA produced through pyruvate dehydrogenase, to produce $\mathrm{C} 4$ moieties. This route for production of $\mathrm{C} 4$ moieties is pricey, compared to anaplerotic $\mathrm{HCO}_{3}$ fixation, in terms of enzymatic cost and carbon loss under mixotrophic condition, as reflected by the objective value and net stoichiometry in Tables 1, 2, respectively. Thus, mixotrophic condition with $\mathrm{HCO}_{3}$ (i.e., +BIC+GLY) was regarded as the optimal condition for lipid production.

\subsubsection{Lipid Demand Variation Analysis}

To further investigate the response of the system to varying lipid demand under mixotrophic condition in the presence of $\mathrm{HCO}_{3}$, LP in Equation (1) was solved repeatedly with incremented demand for lipid production, $v_{\text {txTAG }}$, until no feasible solution was possible, as described in Poolman et al. (2009, 2013) and Villanova et al. (2017). An upper limit was set on glycerol uptake $\left(v_{\text {txGlycerol }} \leq \max G\right)$ and photon flux $\left(v_{v} \leq \max P\right)$ as a proxy for maximum glycerol uptake and light limitation, respectively, and on rubisco reaction $\left(v_{\text {Carboxylase }}+v_{\text {Oxygenase }} \leq \max C\right)$ as proxy for saturation of the Calvin cycle, as in Villanova et al. (2017).

A total of 108 reactions showed variation over the range of imposed lipid demand. Figure 1 shows the reaction responses to increase in lipid demand. Other reactions varying over the lipid demand followed one or other pattern in Figure 1. The change in the metabolic response (regions A, B, C, and D) adjusted with the saturation of glycerol uptake, photon flux, and flux in rubisco reaction. Region A marks low lipid demand and is solely met through glycerol uptake and anaplerotic $\mathrm{HCO}_{3}$ fixation. In region $\mathrm{B}$, flux in glycerol uptake saturates because of the imposed upper limit. The increasing demand for carbon is met through increased flux in $\mathrm{CO}_{2}$ fixation through the Calvin cycle and anaplerotic $\mathrm{HCO}_{3}$ fixation. In region $\mathrm{C}$, flux in photon uptake saturates. Thus, the increased energy demand for increasing lipid production is met through increased flux in mitochondrial electron transport chain. In region $\mathrm{D}$, flux in rubisco reaction saturates. The increasing demand for carbon is met through increased flux in anaplerotic $\mathrm{HCO}_{3}$ fixation through $\mathrm{PEP} /$ pyruvate carboxylase. This region also marks the production of maximum $\mathrm{O}_{2}(\approx 31 \mathrm{nmol} / \mathrm{mL} / \mathrm{min})$, which is in range of experimentally observed $\mathrm{O}_{2}$ evolution under mixotrophic condition with $\mathrm{HCO}_{3}$, as shown in Figure $4(1 \mathrm{nmol} / \mathrm{mL} / \mathrm{min} \approx$ $0.004-0.0085 \mathrm{nmol} / \mathrm{mg} \mathrm{Chl} / \mathrm{min}$ ). Overall, the optimal metabolic state to meet the high lipid demand utilizes glycerol metabolism, light reactions, mitochondrial electron transport chain, the Calvin cycle, and anaplerotic $\mathrm{HCO}_{3}$ fixation.

\subsection{Experimental Analysis}

\subsubsection{Optimization of the ESAW Medium}

Elemental composition of E10 medium was optimized based on the elemental balancing of biomass and medium composition. This is a powerful method to improve the biomass yield of microalgae as shown in other strains (Mandalam and Palsson, 1998; Takács et al., 2007; Harun et al., 2010). This analysis revealed that E10 is not only deficient in 
A
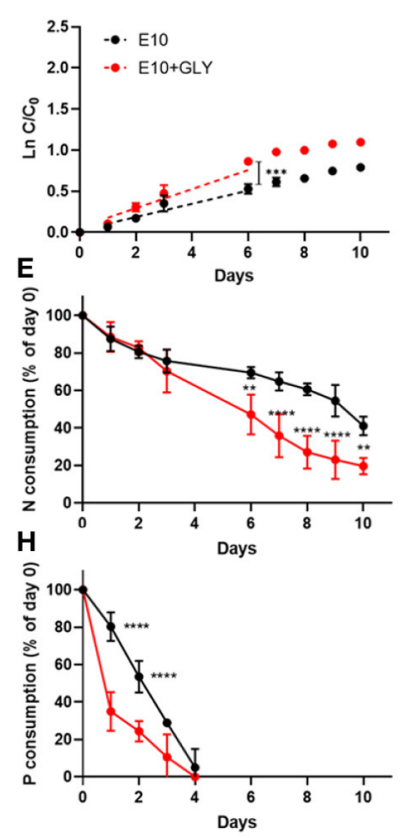

B
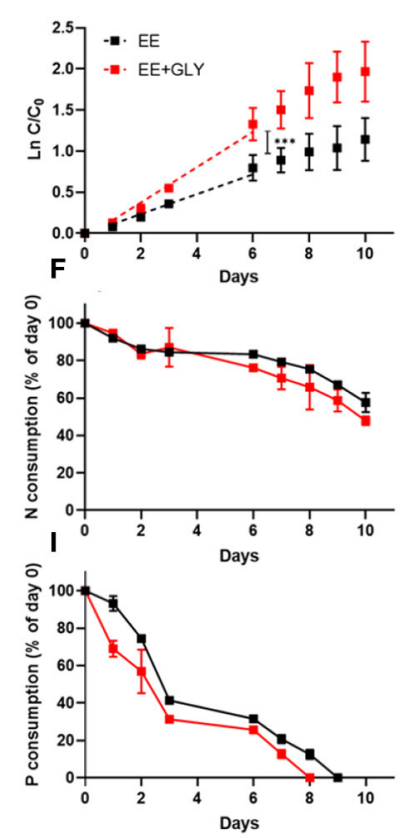

C
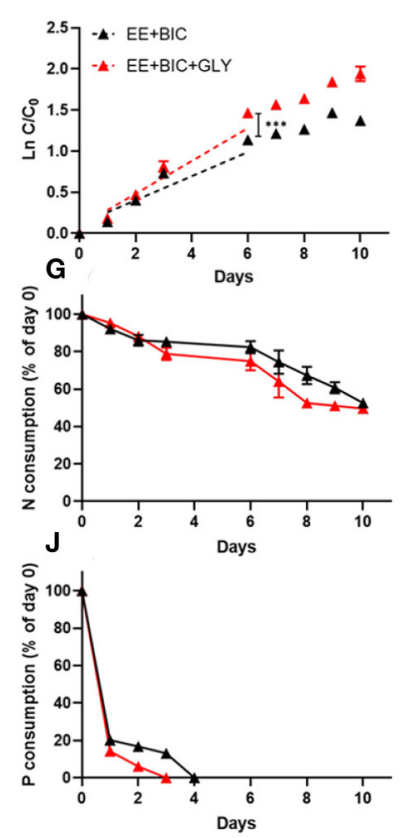

D

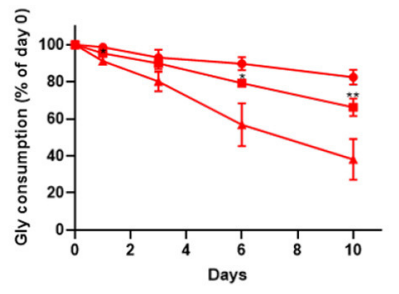

FIGURE 2 | Growth curve, glycerol, nitrate, and phosphate consumption in the initial medium E10 (circle), optimized medium EE (square), and EE+BIC (triangle) in phototrophy (black) and in mixotrophy (red) conditions. Log plot of growth curves in (A) E10, (B) EE, (C) EE+BIC in phototrophy and mixotrophy conditions. C is the biomass concentration expressed in $\mathrm{g} / \mathrm{L}$ at any time and $\mathrm{C} 0$ is the initial biomass concentration. Dotted lines represent the regression curve during the exponential phase of growth (1-6 days). The t-test performed on the slopes were significant with $p$-value (**p $<0.001)$. (D) Glycerol consumption in E10+GLY, EE+GLY, and $\mathrm{EE}+\mathrm{BIC}+\mathrm{GLY}$. Nitrate consumption expressed as \% of day 0 in (E) E10, (F) EE, (G) EE+BIC and phosphate consumption in (H) E10, (I) EE, and (J) EE+BIC. The nutrient consumption was compared in mixotrophy and phototrophy growth in the different medium using two-way ANOVA. Data were considered significant for $p$-values $\left({ }^{\star \star \star \star} P<0.0001\right)$ and $\left.{ }^{* \star} p<0.01\right)$. Each point expressed as mean \pm stdev $(n=4)$ except for + BIC treatments where $n=2$. E10, ESAW 10XN,P; EE, ESAW enriched; EE+BIC, ESAW enriched + bicarbonate; GLY, glycerol.

macronutrients (i.e., nitrogen and phosphorus) but mostly in micronutrients (i.e., iron, manganese, zinc, and copper). Thus, a new medium (called ESAW enriched [EE]) was redesigned by increasing the concentration of $\mathrm{NaNO}_{3}, \mathrm{NaH}_{2} \mathrm{PO}_{4} \cdot \mathrm{H}_{2} \mathrm{O}$, FeEDTA, $\mathrm{ZnSO}_{4} .7 \mathrm{H}_{2} \mathrm{O}, \mathrm{MnSO}_{4} \cdot 4 \mathrm{H}_{2} \mathrm{O}$, and $\mathrm{CuSO}_{4} \cdot 5 \mathrm{H}_{2} \mathrm{O}$ to $2.5 \mathrm{~g} / \mathrm{L}, 0.20 \mathrm{~g} / \mathrm{L}, 0.0216 \mathrm{~g} / \mathrm{L}, 0.589 \mathrm{mg} / \mathrm{L}, 2.55 \mathrm{mg} / \mathrm{L}$, and $0.66 \mathrm{mg} / \mathrm{L}$, respectively. The amount of other elements were maintained the same as E10 medium. EE composition is provided in Supplementary File 2.

Based on the model observations in section 3.1, the EE medium under phototrophic and mixotrophic (with glycerol as organic carbon source) condition was supplemented with $\mathrm{NaHCO}_{3}(1.26 \mathrm{~g} / \mathrm{L})$ to improve the algal growth capacity (i.e., EE+BIC: EE supplemented with bicarbonate and EE+BIC+GLY: EE supplemented with bicarbonate and glycerol, respectively).

\subsubsection{Effect of Glycerol, Bicarbonate, and Nutrients on Phaeodactylum Biomass at Small Scale}

During the exponential phase (1-6 days), the growth rate was significantly increased in mixotrophy compared to phototrophy in each medium $(p<0.001)$ (Figures 2A-C). The exponential growth rate of each condition, the results of $t$-test analysis, and final biomass concentration are shown in Table 3. In addition, EE medium, described in section 3.2.1, in 50-mL Erlenmeyer flasks, increased growth rate by a factor of 1.85 and 1.5 under mixotrophic and phototrophic regime, respectively, as compared to initial E10 medium, without changing the cell dimension. The enhanced mixotrophic growth observed with EE was also mainly due to the positive effect caused by the trace elements (Supplementary Figure 1). The positive effect of the EE medium on mixotrophic growth became even larger upon the addition of bicarbonate in the medium increasing both growth and glycerol consumption (Figures 2C,D and Table 3).

Nitrate and phosphate consumption were also compared in mixotrophic and phototrophy in the different medium (Figures 2E-J). Only in the E10 medium, characterized by lower initial nitrate and phosphate concentration then EE, the consumption of these nutrients was significantly ( $p$-value $<0.01$ ) enhanced in mixotrophy (Figures 2E,H). Phosphate is consumed very rapidly but this does not arrest the growth rate probably because of the polyphosphate accumulation mechanism (Martin et al., 2014; Abida et al., 2015).

On the last day (i.e., day 10) of the experiment, the biomass was surveyed combining photosynthesis, pigment, and lipid analysis (Figures 3A-D). The photosynthesis performances and the photoprotection mechanisms were studied by the determination of the parameters Fv/Fm and NPQ, respectively (as described in section 2.2.1). The data showed that the glycerol 
TABLE 3 | Exponential growth rate, $t$-test analysis, and final biomass concentration in the different media.

\begin{tabular}{|c|c|c|c|c|}
\hline \multirow[t]{2}{*}{ Condition } & \multirow[t]{2}{*}{ Exponential growth rate $\left(\mathrm{d}^{-1}\right)$} & \multicolumn{2}{|c|}{$T$-test analysis } & \multirow[t]{2}{*}{ Final biomass concentration $(g / L)$} \\
\hline & & Phototrophy vs. Mixotrophy & Media vs. Improved media & \\
\hline E10 & $0.08 \pm 0.02$ & E10 vs. E10+GLY $\left.{ }^{(* \star \star}\right)$ & E10 vs. EE $\left(^{\star \star \star \star}\right)$ & $0.874 \pm 0.075$ \\
\hline E10+GLY & $0.116 \pm 0.04$ & & 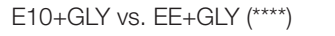 & $1.177 \pm 0.059$ \\
\hline EE & $0.121 \pm 0.008$ & EE vs. EE+GLY $\left({ }^{\star \star \star \star}\right)$ & EE vs. EE+BIC $\left(^{*}\right)$ & $1.095 \pm 0.027$ \\
\hline $\mathrm{EE}+\mathrm{GLY}$ & $0.215 \pm 0.05$ & & $\mathrm{EE}+\mathrm{GLY}$ vs. EE+BIC+GLY (ns) & $1.857 \pm 0.104$ \\
\hline $\mathrm{EE}+\mathrm{BIC}$ & $0.147 \pm 0.013(n=2)$ & $\mathrm{EE}+\mathrm{BIC}$ vs. EE+BIC+GLY $\left(^{\star \star \star \star}\right)$ & & $1.306 \pm 0.117$ \\
\hline $\mathrm{EE}+\mathrm{BIC}+\mathrm{GLY}$ & $0.2 \pm 0.08(n=2)$ & & & $2.017 \pm 0.032$ \\
\hline
\end{tabular}

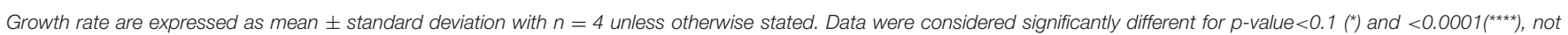
significant (ns): $p$-value $>0.1$.

A

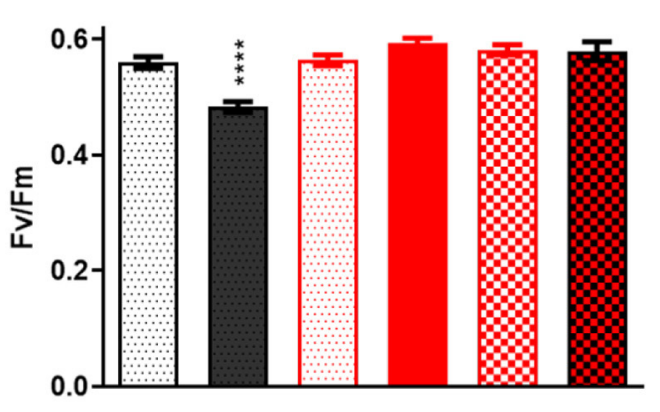

B

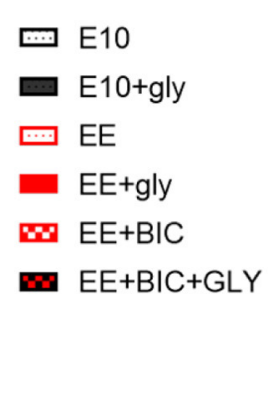

D

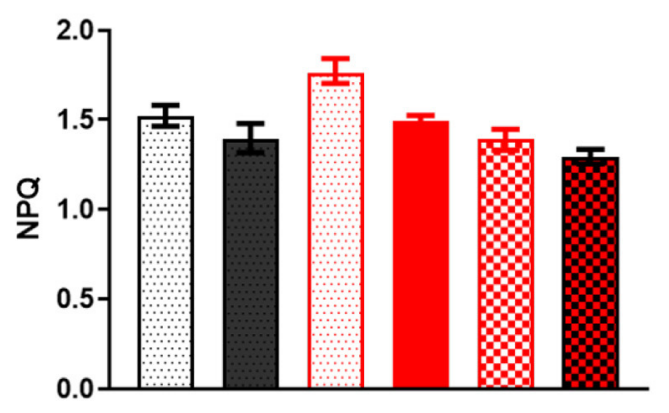

C

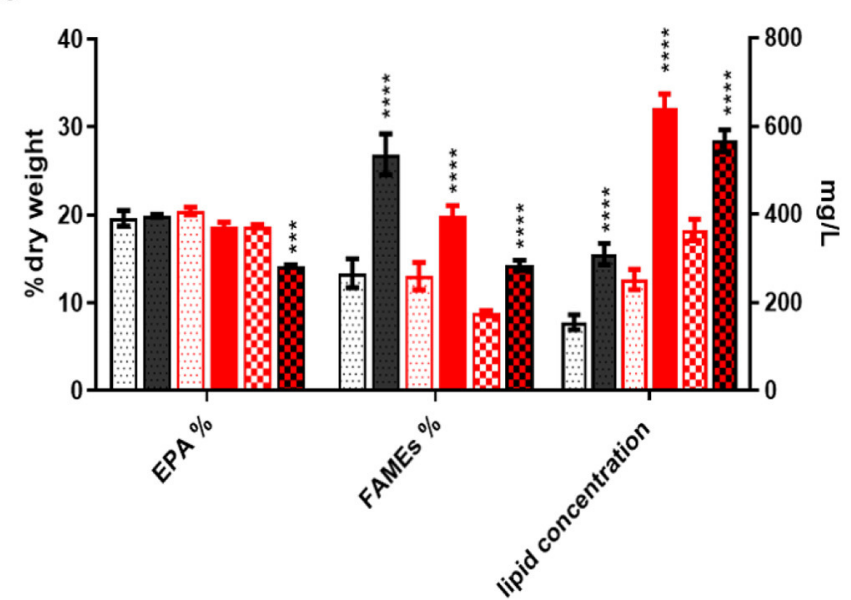

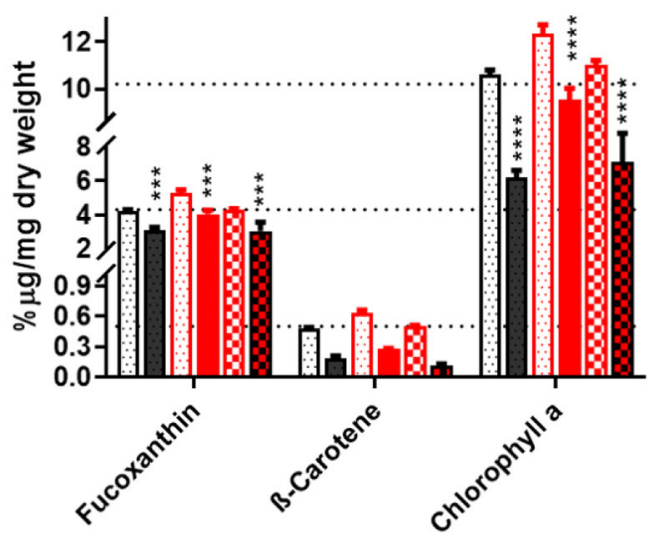

FIGURE 3 | Biomass analysis and photosynthetic parameters analysis. (A) Fv/Fm and (B) NPQ measurement, (C) Lipids, and (D) pigments analysis in the initial medium E10 and in the optimized medium EE and EE+BIC in both mixotrophy and phototrophy. In (C), right $Y$-axis (mg/L) is for lipid concentration while the left Y-axis(\%) is for FAMES and EPA. One-way analysis of variance (ANOVA) test was applied in order to compare the phototrophy and mixotrophy in different media. Data were considered significant for $p$-values $\left({ }^{\star \star \star *} p<0.0001\right)$ and $\left({ }^{\star \star *} p<0.001\right)$. Each point expressed as mean \pm stdev $(n=4)$. E10, ESAW 10XN,P; EE, ESAW enriched; EE+BIC, ESAW enriched + bicarbonate; GLY, glycerol.

decreases the photosynthesis performance only in E10 ( $p$-value $<0.0001$ ), most likely due to the rapid consumption of the $\mathrm{N}$ (Figures 2D, 3A). No significant differences were detected in photoprotection in the tested conditions (Figure 3B).

The lipid analysis was divided into eicosapentaenoic acid (EPA), fatty acid, and total lipid concentration (Figure 3C). The
EPA concentration was similar in all the tested conditions with the exception of $\mathrm{EE}+\mathrm{BIC}+\mathrm{GLY}$ where the concentration was lower $(p$-value $<0.001)$. The glycerol enhanced the fatty acid content compared to their phototrophic counterpart ( $p$-value $<0.0001$ ) and its effect was higher in E10+GLY, possibly due to $\mathrm{N}$ starvation that trigger lipid production. In EE, which had 


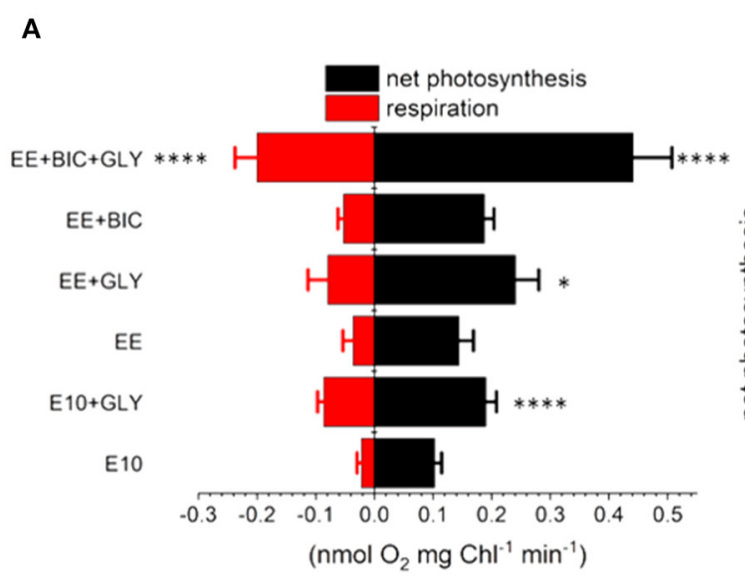

B

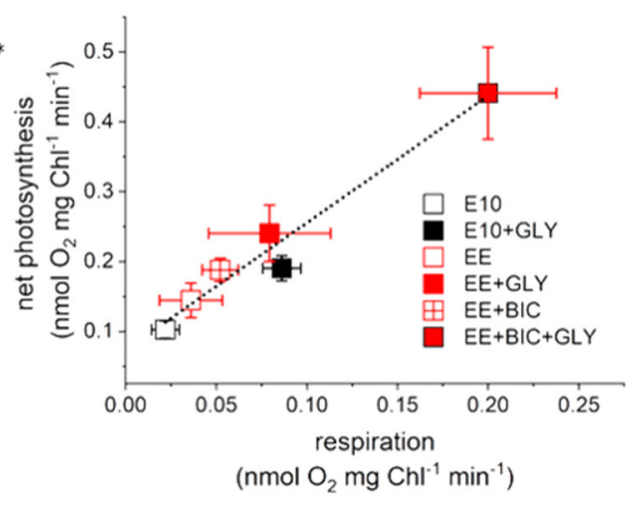

FIGURE 4 | (A) Direct assessment of oxygen consumption/production by a polarographic approach in E10, EE, and EE + BIC in both phototrophy and mixotrophy (+GLY). Measurements were done in the light (i.e., photosynthesis, black) and in the dark (i.e., respiration, red). One-way analysis of variance (ANOVA) test was applied in order to compare the phototrophy and mixotrophy in different media. Data were considered significant for $p$-values $<0.1\left({ }^{\star \star * *} p<0.0001,{ }^{\star} p<0.1\right)$.

(B) Relationship between oxygen uptake and net photosynthesis measured with a Clark electrode. Dotted lines represent a fitted regression. Each point expressed as mean \pm stdev $(n=4-12)$.

higher nitrogen concentration, the glycerol mostly increased the total lipid concentration (Figure 3C, $p$-value $<0.0001$ ). In addition, the bicarbonate increased the total lipids concentration, $p$-value $<0.0001$, when compared to E10 and EE, under phototrophic conditions.

EE also increased the concentration of pigments, especially fucoxanthin, and chlorophyll A, as shown in Figure 3D, compared to E10 in both phototrophy and mixotrophy conditions with $p$-value $<0.01-0.0001$. The mixotrophy, however, decreased the concentration of pigments in all the conditions as compared to their phototrophic counterparts with $p$-value $<0.001-0.0001$, but this is less evident in EE+GLY that possess a higher concentration of nitrate and phosphate on the day of the analysis (i.e., day 10; Figures 2C,D). No statistically significant difference was observed in $\beta$-carotene under different conditions. However, decrease of carotenoids and chlorophyll in mixotrophy has been shown in P. tricornutum (Liu et al., 2009 b), but also the nutrients limitation has a key role in the degradation of pigments (Alipanah et al., 2015, 2018). Data for all flask experiments are available in Supplementary File 3.

Finally, respiration and photosynthesis rates were measured as $\mathrm{O}_{2}$ exchange rates using a Clark-type oxygen electrode with an in vivo experiment at $19^{\circ} \mathrm{C}$ (Hansatech Instruments) (as described in section 2.2.2). As shown in Figure 4A, the glycerol enhanced the respiration rates in all the tested conditions ( $p$-value $<0.1-0.0001$ ), confirming previous results (Grama et al., 2015). The oxygen consumption was higher in the optimized medium (i.e., both $\mathrm{EE}$ and $\mathrm{EE}+\mathrm{BIC}$ ), as expected based on the finding that glycerol consumption and growth performances are higher in these conditions (Figure 2). Net photosynthesis (calculated as oxygen evolution corrected by dark respiration) was also increased by glycerol, in a way that was commensurate to the respiration enhancement (Figure 4B). Indeed, we found a linear relationship between the photosynthetic and respiratory performances. Overall these results suggest that mixotrophy enhances respiration (via glycerol consumption) and photosynthesis, possibly through energetic exchanges between the two energy making organelles in line with earlier hypotheses (Bailleul et al., 2015).

\subsubsection{Scale-Up in 2-L Photobioreactor}

The experiment was further upscaled to 2-L photobioreactors, as specified in section 2.1.2, in E10, EE, and EE+bicarbonate under both phototrophic and mixotrophic condition. Photobioreactors with mixotrophic culture and $\mathrm{EE}+\mathrm{BIC}$ condition were supplemented with 4.6 and $1.26 \mathrm{~g} / \mathrm{L}$ of glycerol and $\mathrm{NaHCO}_{3}$, respectively, at a regular time interval. Since $\mathrm{HNO}_{3}$ was also used to regulate the $\mathrm{pH}$ of the culture, as mentioned in section 2.1.2, the cultures were never deficient in terms of nitrogen while $\mathrm{NaH}_{2} \mathrm{PO}_{4}$ was added to the culture as needed. However, in the samples E10 and E10+GLY the $\mathrm{pH}$ was regulated by the addition of $0.4 \mathrm{~N}$ of $\mathrm{H}_{2} \mathrm{SO}_{4}$ to maintain the original $\mathrm{N}$ concentration of the medium.

As shown in Table 4 and Figure 5, exponential growth rate was largely increased in optimized medium (i.e., EE and $\mathrm{EE}+\mathrm{BIC}$ ) under mixotrophic condition when compared to initial conditions (E10). The growth rates in flask experiments (section 3.2.2 and Table 3 and bioreactor (Table 4) were highly comparable for equivalent conditions, and showed the same pattern of effects for medium supplementation and mixotrophy. The biomass concentration and biomass, lipid (as fatty acids and EPA), carbohydrates, and fucoxanthin productivities were compared in the different conditions and are summarized in Table 4. EE $+\mathrm{BIC}+\mathrm{GLY}$ proves to be the best condition. The biomass concentration is increased by a factor of about 9 comparing to the initial conditions E10. Pigment, fatty acid, EPA, and carbohydrate also increases with $p$-value $<0.0001$ (Supplementary File 4), when compared to initial conditions 
TABLE 4 | Growth rate, final biomass concentration, maximum FAMEs, EPA, fucoxanthin, and carbohydrate productivity in the different media, E10, EE, and EE+BIC under phototrophic and mixotrophic conditions in 2-L photobioreactor.

\begin{tabular}{|c|c|c|c|c|c|c|}
\hline & E10 & E10+GLY & EE & $\mathrm{EE}+\mathrm{GLY}$ & $\mathrm{EE}+\mathrm{BIC}$ & $\mathrm{EE}+\mathrm{BIC}+\mathrm{GLY}$ \\
\hline Growth rate, $\left(\mathrm{d}^{-1}\right)$ & $0.078 \pm 0.004(n=5)$ & $0.098 \pm 0.004(n=6)$ & $0.06 \pm 0.01(n=6)$ & $0.19 \pm 0.02$ & $0.16 \pm 0.02(n=6)$ & $0.18 \pm 0.03(n=5)$ \\
\hline Final biomass conc, (g/L) & $1.32 \pm 0.08(n=6)$ & $1.46 \pm 0.08(n=6)$ & $1.6 \pm 0.14(n=6)$ & $5.03 \pm 0.19(n=6)$ & $2.58 \pm 0.15(n=6)$ & $11.55 \pm 0.24$ \\
\hline max FAMEs, (mg/L/d) & $14.59 \pm 1.12$ & $32.45 \pm 2.22$ & $9.98 \pm 1.78$ & $23.80 \pm 2.86$ & $21.61 \pm 3.39$ & $51.96 \pm 0.61$ \\
\hline $\max$ EPA, (mg/L/d) & $2.12 \pm 0.08$ & $2.87 \pm 0.21$ & $1.40 \pm 0.14$ & $3.98 \pm 0.51$ & $3.2 \pm 1.7$ & $9.51 \pm 0.13$ \\
\hline max Fucoxanthine, (mg/L/d) & $0.36 \pm 0.05$ & $0.21 \pm 0.11$ & - & - & $0.71 \pm 0.06$ & $1.97 \pm 0.34$ \\
\hline max Carbohydrate, (mg/L/d) & $16.76 \pm 1.35$ & $31.03 \pm 2.61$ & $4.95 \pm 0.54$ & $25.50 \pm 2.06$ & $16.85 \pm 13.18$ & $54.91 \pm 2.40$ \\
\hline
\end{tabular}

Results are expressed as mean \pm stdev with $n=4$ unless otherwise stated.
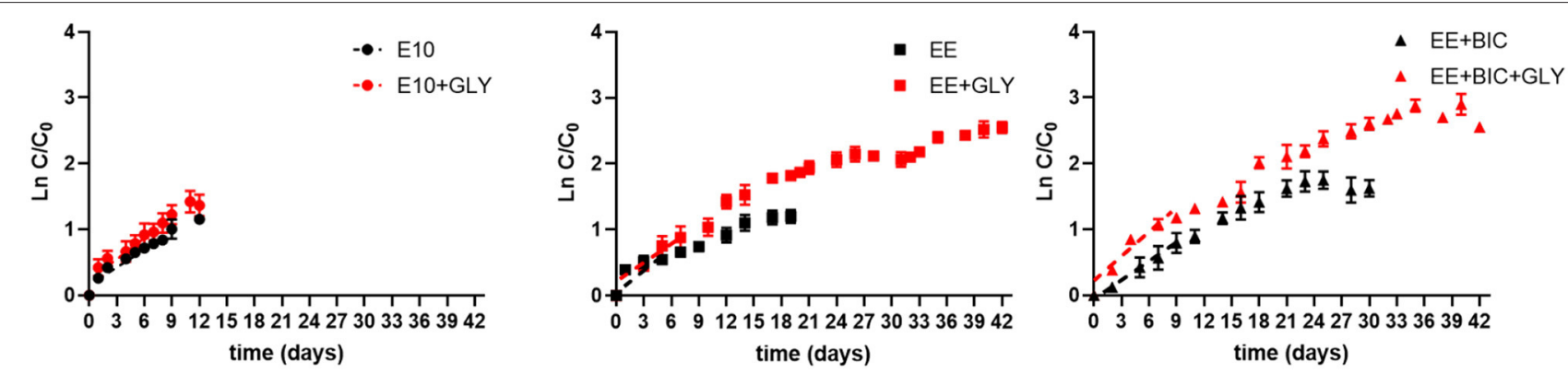

FIGURE 5 | Scale-up to photobioreactors. Log plot of growth curves in 2-L fermentor in E10 (circle), EE (square), and EE+ BIC (triangle) in both phototrophic (black) and mixotrophic (red) modes. $C$ is the biomass concentration expressed in $\mathrm{g} / \mathrm{L}$ at any time, and CO is the initial biomass concentration. Dotted lines represent the regression curve during the exponential phase of growth (1-6 days). The slopes of the curve (growth rates) of each media under phototrophy and mixotrophy conditions are shown in Table 4. Results are expressed as mean \pm stdev with $n$ values as stated in Table 4.

(E10). Higher lipids, especially fatty acids, were shown in E10 \pm GLY compared to EE \pm GLY, most likely because of the N limitation in E10 media that triggers lipid storage. Our result demonstrates that the light and supplementation of bicarbonate induced higher production of lipids even without $\mathrm{N}$ limitation overcoming the trade-off between growth and lipid production.

\section{DISCUSSION AND CONCLUSION}

In this study, we have combined metabolic modeling and experimental approach to design an optimized growth media (called EE) for P. tricornutum. The model analysis suggested contribution of glycerol and $\mathrm{HCO}_{3}$ fixation toward lipid production. Further, it suggest that high lipid production utilizes various aspects of metabolism such as photosynthesis, respiration, inorganic $\left(\mathrm{HCO}_{3}\right)$, and organic (glycerol) carbon fixation. The addition of both organic (i.e., glycerol) and inorganic $\mathrm{C}$ (i.e., $\mathrm{NaHCO}_{3}$ ), along with the optimization of the trace elements, improved both biomass quantity and quality in $P$. tricornutum in our study.

Carbon is the major macronutrient that affects the growth of microalgae and to increase the biomass/growth and storage compounds such as lipid and carbohydrate, it is necessary to increase the dissolved inorganic carbon (DIC) concentration, as shown in different algal species, including Phaeodactylum (Levering et al., 2016; Hammer et al., 2019). The most common practice to supplement the algal culture with the DIC is to bubble air into the growth medium. Another method includes addition of $\mathrm{HCO}_{3}$ to the media (Lohman et al., 2015; Mokashi et al., 2016), which is a cheaper and more suitable inorganic carbon alternative to the $\mathrm{CO}_{2}$. Diatoms, including Phaeodactylum, possesses biophysical and/or biochemical $\mathrm{CO}_{2}$ - concentrating mechanism (CCM) (Reinfelder et al., 2000; Roberts et al., 2007; Hopkinson et al., 2011; Matsuda et al., 2011). Biophysical CCMs involve active transport of $\mathrm{CO}_{2}$ or $\mathrm{HCO}_{3}$ and $\mathrm{CA}$ maintains equilibrium between the two species by catalyzing the reversible interconversion of $\mathrm{CO}_{2}$ and water into $\mathrm{HCO}_{3}$ and protons. Biochemical CCMs involving C4-type photosynthesis, on the other hand, utilizes carboxylation enzymes such as $\mathrm{PEP} /$ pyruvate carboxylase, which catalyzes the carboxylation of $\mathrm{PEP} /$ pyruvate with $\mathrm{HCO}_{3}$, forming a $\mathrm{C} 4$ carbon compound. This compound is then cleaved by decarboxylating enzymes to produce $\mathrm{CO}_{2}$ in the proximity of Rubisco (Sage, 2004). In addition, anaplerotic production of $\mathrm{C} 4$ skeletons through $\mathrm{HCO}_{3}$ fixation by $\mathrm{PEP} /$ pyruvate carboxylase independent of photosynthesis, as observed in the model (section 3.1), has also been reported in diatoms (Granum and Myklestad, 1999; Needoba and Harrison, 2004). Results in this study confirm that addition of $\mathrm{HCO}_{3}$ improves the algal growth, although further investigation using $\mathrm{C}$ labeling and multi-omics techniques would be required to confirm the fate and mechanism of $\mathrm{HCO}_{3}$ utilization in $P$. tricornutum.

In addition, most microalgae can simultaneously assimilate organic carbon such as glycerol, fructose, glucose, lactose, 
mannose, and acetate (Garcì et al., 2004; Villanova et al., 2017). $P$. tricornutum is also able to use organic carbon in presence of light, and in particular, the glycerol has been shown to be the best candidate for enhancing biomass and lipids productivity (Garcia et al., 2005, 2006, 2013; Villanova et al., 2017). Model analysis shows that glycerol enters the central carbon metabolism through glycolysis and PPP and that both the Calvin cycle located in plastid and PPP located in cytosol can be active simultaneously. In other photosynthetic eukaryotes where both the Calvin cycle and PPP are located in the same compartment (i.e., plastid), the simultaneous operation of the Calvin cycle and oxidative limb of PPP would lead to the futile cycling of NADPH. This is prevented by the action of the thioredoxin system (Anderson, 1981, 1986; Sibley and Anderson, 1989; Schurmann and Jacquot, 2000). This is a redox mediated system that serves (among other things) to activate the oxidative limb of the PPP in the dark, and inactivate it in the light. Although diatoms possess the thioredoxin system, its targets are unclear: the only Calvin cycle enzyme under thioredoxin control appears to be fructose bisphosphate aldolase (Wilhelm et al., 2006; Kroth et al., 2008). Moreover, PPP is translocated to the cytosol. Overall, we propose that this compartmental re-arrangement and redox deregulation could possibly be a metabolic advantage for $P$. tricornutum to simultaneously activate both the processes.

The increased concentration of micronutrient has also been shown to enhance both biomass and biotech relevant molecules, as TAGs, probably due their involvement in the key enzymes of photosynthesis, respiration, and carbon fixation in microalgae (Morel et al., 2003). Here, we showed that the addition of both organic (i.e., glycerol) and inorganic carbon (i.e., $\mathrm{NaHCO}_{3}$ ), along with increased micronutrients (trace elements), improved both biomass quantity and quality in P. tricornutum. It also enhanced both the respiration and photosynthesis performance (both in the model analysis and experimental results), suggesting that there is an energetic coupling between chloroplast and mitochondria and the communication between the two organelles is crucial for optimizing carbon fixation and growth as reported by Bailleul et al. (2015).

The medium optimization by implementing the micronutrients and $\mathrm{NaHCO}_{3}$ supply largely enhanced mixotrophy growth, allowing to reach the state of the art biomass and lipid concentration levels. The total lipid and biomass concentration in our improved EE medium, under mixotrophic regime in the flask experiments, are higher $(641 \mathrm{mg} / \mathrm{L}$ and $1.8 \mathrm{~g} / \mathrm{L}$, respectively) as compared to previous experiments by Yang et al. (2017) and Yodsuwan et al. (2017) in f/2 medium, which obtained total lipid and biomass concentration in the range of $40-133 \mathrm{mg} / \mathrm{L}$ and $0.2-0.4 \mathrm{~g} / \mathrm{L}$, respectively, for the same or higher duration of the cultivation time. Fucoxanthin concentration in EE medium is comparable to that in $f / 2$ medium (4.47 mg/g DW) (Yang et al., 2017). In upscaled 2-L photobioreactor, Fucoxanthin concentration was increased by a factor of about 6 compared to the initial condition. The biomass concentration, which was achieved in the presence of relatively low light intensities (in the range of $70-300 \mu \mathrm{E} \mathrm{m}^{-2} \mathrm{~s}^{-1}$ ) is comparable to previous experiments by Garcia et al. (2013) that used light intensity of $750 \mu \mathrm{E} \mathrm{m}^{-2} \mathrm{~s}^{-1}$ where similar biomass concentration was obtained, in comparable cultivation time, in both phototrophy and mixotrophy (5 and $14 \mathrm{~g} / \mathrm{L}$, respectively). A further investigation would be required to comment if increasing light intensity, as in Garcia et al. (2013), would have further enhanced the biomass and lipid concentration in our improved media.

The addition of glycerol also enhances carbohydrates concentration in P. tricornutum (Villanova et al., 2017). Here, the carbohydrate productivity was enhanced of about 3 times compared to the initial condition. The inhibition of the biosynthesis of storage carbohydrates could potentially direct the carbon (derived from glycerol) toward TAG production as already reported in the case of the main sugar storage polymer, chrysolaminarin in Daboussi et al. (2014). Our study demonstrated that the combination of different optimization processes, i.e., elemental balancing, process design and mathematical model, can be successfully integrated to design an optimized growth media that, in our experiments, have increased the algal production capabilities. Moreover, the algal productivity and lipid production could be further enhanced by metabolic engineering and improving the quality and quantity of light.

\section{DATA AVAILABILITY STATEMENT}

The datasets presented in this study can be found in online repositories. GSM is available from BioModels repository with model ID MODEL2102080001 (www.ebi.ac.uk/biomodels/). GSM (in ScrumPy and SBML format) along with python scripts to generate the results reported in this study is available from https://gitlab.com/singhdi/phaeomodel.

\section{AUTHOR CONTRIBUTIONS}

VV, DS, GF, JP, DF, AL, and MP conceived and designed the experiments, and analyzed data. VV and DS performed experiments and wrote the manuscript. DS and MP performed the model simulation. All the authors approved the final version of the manuscript before the submission.

\section{FUNDING}

This project was funded by the European Commission-Marie Curie Initial Training Network Accliphot (FP7-PEPOPLE2012-ITN; 316427) and all authors gratefully acknowledge the support of the Marie Curie Initial Training Network Accliphot. The publication fee was funded by the European Union's Horizon 2020 research and innovation program under the Marie Skłodowska-Curie grant agreement No. 844909. VV is grateful for financial support from the European Commission through the Marie Skłodowska-Curie Individual Fellowships (H2020MSCA-IF-2018, grant: 844909). DS gratefully acknowledge the support of the Biotechnology and Biological Sciences Research 
Council (BBSRC) through the BBSRC Institute Strategic Programme Microbes in the Food Chain BB/R012504/1.

\section{ACKNOWLEDGMENTS}

We would like to sincerely thank Prof. Ross Carlson from Montana State University for providing GSM of $P$. tricornutum which served as the basis for the model development. We also sincerely thank the laboratory of analysis and fermentation at Fermentals S.A. for their help on the experiment.

\section{SUPPLEMENTARY MATERIAL}

The Supplementary Material for this article can be found online at: https://www.frontiersin.org/articles/10.3389/fpls.2021. 642199/full\#supplementary-material

\section{REFERENCES}

Abida, H., Dolch, L.-J., Meï, C., Villanova, V., Conte, M., Block, M. A., et al. (2015). Membrane glycerolipid remodeling triggered by nitrogen and phosphorus starvation in Phaeodactylum tricornutum. Plant Physiol. 167, 118136. doi: 10.1104/pp.114.252395

Alipanah, L., Rohloff, J., Winge, P., Bones, A., and Brembu, T. (2015). Whole-cell response to nitrogen deprivation in the diatom Phaeodactylum tricornutum. J. Exp. Bot. 66, 6281-6296. doi: 10.1093/jxb/erv340

Alipanah, L., Winge, P., Rohloff, J., Najafi, J., Brembu, T., and Bones, A. (2018). Molecular adaptations to phosphorus deprivation and comparison with nitrogen deprivation responses in the diatom Phaeodactylum tricornutum. PLoS ONE 13:e0193335. doi: 10.1371/journal.pone.0193335

Anderson, L. E. (1981). Light inactivation of transaldolase in pea leaf chloroplasts. Biochem. Biophys. Res. Commun. 99, 1199-1202. doi: 10.1016/0006-291X(81)90746-4

Anderson, L. E. (1986). Light/dark modulation of enzyme activity in plants. $A d v$. Bot. Res. 12, 1-46. doi: 10.1016/S0065-2296(08)60192-5

Annett, A., Lapi, S., Ruth, T., and Maldonado, M. (2008). The effects of Cu and Fe availability on the growth and $\mathrm{Cu}: \mathrm{C}$ ratios of marine diatoms. Limnol. Oceanogr. 53, 2451-2461. doi: 10.4319/lo.2008.53.6.2451

Armbrust, E. (2009). The life of diatoms in the world's oceans. Nature 459, 185-192. doi: 10.1038/nature08057

Bailleul, B., Berne, N., Murik, O., Petroutsos, D., Prihoda, J., Tanaka, A., et al. (2015). Energetic coupling between plastids and mitochondria drives Co2 assimilation in diatoms. Nature 524, 366-369. doi: 10.1038/nature14599

Berges, J. A., Franklin, D. J., and Harrison, P. J. (2001). Evolution of an artificial seawater medium: improvements in enriched seawater, artificial water over the last two decades. J. Phycol. 37, 1138-1145. doi: 10.1046/j.1529-8817.2001.01052.x

Blaby-Haas, C., and Merchant, S. (2017). Regulating cellular trace metal economy in algae. Curr. Opin. Plant Biol. 39, 88-96. doi: 10.1016/j.pbi.2017.06.005

Chisti, Y. (2007). Biodiesel from microalgae. Biotechnol. Adv. 25, 294-306. doi: 10.1016/j.biotechadv.2007.02.001

Chisti, Y. (2008). Biodiesel from microalgae beats bioethanol. Trends Biotechnol. 26, 126-131. doi: 10.1016/j.tibtech.2007.12.002

Choudhary, M., Jetley, U., Khan, M., Zutshi, S., and Fatma, T. (2007). Effect of heavy metal stress on proline, malondialdehyde, and superoxide dismutase activity in the cyanobacterium Spirulina platensis-s5. Ecotoxicol. Environ. Saf. 66, 204-209. doi: 10.1016/j.ecoenv.2006.02.002

Daboussi, F., Leduc, S., Maréchal, A., Dubois, G., Guyot, V., PerezMichaut, C., et al. (2014). Genome engineering empowers the diatom Phaeodactylum tricornutum for biotechnology. Nat. Commun. 5:3831. doi: $10.1038 /$ ncomms4831
Supplementary File 1 | Calibration curve obtained by the correlation of the absorbance and dry weight.

Supplementary File 2 | EE medium composition.

Supplementary File 3 | All experimental data and statistical analysis for flask experiments.

Supplementary File 4 | All experimental data and statistical analysis for 2-L photobioreactor experiments.

Supplementary Figure 1 | Optimization of growth medium in mixotrophic condition. (A) Growth curve (from single experiment) and (B) pictures of flasks in the last day of cultures in the initial medium E10 (black line) in E10+N,P (green line), E10+Fe (yellow line), E10+Me (blue line), and in the optimized medium EE (red line). E10, ESAW 10XN,P; E10+ N,P, ESAW 10XN,P + enriched concentration of $\mathrm{N}$ and $\mathrm{P} ; \mathrm{E} 10+\mathrm{Fe}$, ESAW 10XN,P + enriched concentration of Fe; E10+Me, ESAW 10XN,P + enriched concentration of $\mathrm{Cu}, \mathrm{Zn}$, and $\mathrm{Mn}$; EE,

ESAW enriched.

d’Ippolito, G., Sardo, A., Paris, D., Vella, F., Adelfi, M. G., Botte, P., et al. (2015). Potential of lipid metabolism in marine diatoms for biofuel production. Biotechnol. Biofuels 8:28. doi: 10.1186/s13068-015-0212-4

Eriksen, N. (2008). The technology of microalgal culturing. Biotechnol. Lett. 30, 1525-1536. doi: 10.1007/s10529-008-9740-3

Fabris, M., Matthijs, M., Rombauts, S., Vyverman, W., Goossens, A., and Baart, G. J. E. (2012). The metabolic blueprint of Phaeodactylum tricornutum reveals a eukaryotic Entner-Doudoroff glycolytic pathway. Plant J. 70, 1004-1014. doi: 10.1111/j.1365-313X.2012.04941.x

Falkowski, P. G., and Owens, T. G. (1980). Light-shade adaptation. Plant Physiol. 66, 592-595. doi: 10.1104/pp.66.4.592

Fell, D. A., and Small, R. J. (1986). Fat synthesis in adipose tissue. An examination of stoichiometric constraints. Biochem. J. 238, 781-786. doi: 10.1042/bj2380781

Folch, J., Lees, M., and Stanley, G. H. S. (1957). A simple method for the isolation and purification of total lipides from animal tissues. J. Biol. Chem. 226, 497-509. doi: 10.1016/S0021-9258(18)64849-5

Garcì, M., Sevilla, J. M. F., Fernández, F. G. A., Grima, E. M., and Camacho, F. G. (2004). Mixotrophic growth of Phaeodactylum tricornutum on glycerol: growth rate and fatty acid profile. J. Appl. Phycol. 12, 239-248. doi: 10.1023/A:1008123000002

Garcìa, M. D. C. C., Miròn, A. S., Sevilla, J. M. F., Grima, E. M., and Camacho, F. G. (2005). Mixotrophic growth of the microalga Phaeodactylum tricornutum: influence of different nitrogen and organic carbon sources on productivity and biomass composition. Process Biochem. 40, 297-305. doi: $10.1016 /$ j.procbio.2004.01.016

Garcìa, M. D. C. C., Sevilla, J. M. F., Miròn, Antonio Sànchezand Camacho, F. G., Gòmez, A. C., and Grima, E. M. (2006). Mixotrophic production of marine microalga Phaeodactylum tricornutum on various carbon sources. J. Microbiol. Biotechnol. 16:689.

Garcìa, M. D. C. C., Sevilla, J. M. F., Miròn, A. S., Camacho, F. G., Gòmez, A. C., and Grima, E. M. (2013). Mixotrophic growth of Phaeodactylum tricornutum on fructose and glycerol in fed-batch and semi-continuous modes. Bioresour. Technol. 147, 569-576. doi: 10.1016/j.biortech.2013.08.092

Grama, B., Agathos, S., and Jeffryes, C. (2015). Balancing photosynthesis and respiration increases microalgal biomass productivity during photoheterotrophy on glycerol. ACS Sustain. Chem. Eng. 4, 1611-1618. doi: 10.1021/acssuschemeng.5b01544

Granum, E., and Myklestad, S. M. (1999). Effects of NH4+ assimilation on dark carbon fixation and beta-1,3-glucan metabolism in the marine diatom Skeletonema costatum (bacillariophyceae). J. Phycol. 35, 1191-1199. doi: 10.1046/j.1529-8817.1999.3561191.x

Gu, C., Kim, G., Kim, W., Kim, T. Y., and Lee, S. Y. (2019). Current status and applications of genome-scale metabolic models. Genome Biol. 20:121. doi: 10.1186/s13059-019-1730-3 
Guillard, R. R., and Ryther, J. H. (1962). Studies of marine planktonic diatoms. I. Cyclotella nana hustedt, and detonula confervacea (cleve) gran. Can. J. Microbiol. 8, 229-239. doi: 10.1139/m62-029

Guillard, R. R. L. (1975). "Culture of phytoplankton for feeding marine invertebrates," in Culture of Marine Invertebrate Animals, eds W. L. Smith, M. H. Chanley (Boston, MA: Springer). doi: 10.1007/978-1-4615-8714-9_3

Hammer, K. J., Kragh, T., and Sand-Jensen, K. (2019). Inorganic carbon promotes photosynthesis, growth, and maximum biomass of phytoplankton in eutrophic water bodies. Freshw. Biol. 64, 1956-1970. doi: 10.1111/fwb.13385

Harrison, P. J., Waters, R. E., and Taylor, F. J. R. (1980). A broad spectrum artificial sea water medium for coastal and open ocean phytoplankton1. J. Phycol. 16, 28-35. doi: 10.1111/j.0022-3646.1980.00028.x

Harun, R., Halim, R., and Forde, G. (2010). Cultivation medium design via elemental balancing for Tetraselmis suecica. Chem. Biochem. Eng. Q. 24, 361369.

Hildebrand, M., Davis, A. K., Smith, S. R., Traller, J. C., and Raffaela, A. (2012). The place of diatoms in the biofuels industry. Biofuels 3, 221-240. doi: $10.4155 / \mathrm{bfs} .11 .157$

Holzhütter, H.-G. (2006). The generalized flux-minimization method and its application to metabolic networks affected by enzyme deficiencies. Biosystems 83, 98-107. doi: 10.1016/j.biosystems.2005.04.008

Hopkinson, B. M., Dupont, C. L., Allen, A. E., and Morel, F. M. M. (2011). Efficiency of the co2-concentrating mechanism of diatoms. Proc. Natl. Acad. Sci. U.S.A. 108, 3830-3837. doi: 10.1073/pnas.1018062108

Hunt, K. A., Folsom, J. P., Taffs, R. L., and Carlson, R. P. (2014). Complete enumeration of elementary flux modes through scalable demand-based subnetwork definition. Bioinformatics 30, 1569-1578. doi: 10.1093/bioinformatics/btu021

Kim, W. J., Kim, H. U., and Lee, S. Y. (2017). Current state and applications of microbial genome-scale metabolic models. Curr. Opin. Syst. Biol. 2, 10-18. doi: 10.1016/j.coisb.2017.03.001

Kroth, P. G., Chiovitti, A., Gruber, A., Martin-Jezequel, V., Mock, T., Parker, M. S., et al. (2008). A model for carbohydrate metabolism in the diatom Phaeodactylum tricornutum deduced from comparative whole genome analysis. PLoS ONE 3:e1426. doi: 10.1371/journal.pone.0001426

Levering, J., Broddrick, J., Dupont, C. L., Peers, G., Beeri, K., Mayers, J., et al. (2016). Genome-scale model reveals metabolic basis of biomass partitioning in a model diatom. PLoS ONE 11:e0155038. doi: 10.1371/journal.pone. 0155038

Liaud, M.-F., Lichtl, C., Apt, K., Martin, W., and Cerff, R. (2000). Compartmentspecific isoforms of TPI and GAPDH are imported into diatom mitochondria as a fusion protein: evidence in favor of a mitochondrial origin of the eukaryotic glycolytic pathway. Mol. Biol. Evol. 17, 213-223. doi: 10.1093/oxfordjournals.molbev.a026301

Liu, X., Duan, S., Li, A., Xu, N., Cai, Z., and Hu, Z. (2009a). Effects of organic carbon sources on growth, photosynthesis, and respiration of Phaeodactylum tricornutum. J. Appl. Phycol. 21, 239-246. doi: 10.1007/s10811-0089355-z

Liu, X.-J., Duan, S.-S., Li, A.-F., and Sun, K.-F. (2009b). Effects of glycerol on the fluorescence spectra and chloroplast ultrastructure of Phaeodactylum tricornutum (Bacillariophyta). J. Integr. Plant Biol. 51, 272-278. doi: 10.1111/j.1744-7909.2008.00767.x

Lohman, E., Gardner, R., Pedersen, T., Peyton, B., Cooksey, K., and Gerlach, R. (2015). Optimized inorganic carbon regime for enhanced growth and lipid accumulation in Chlorella vulgaris. Biotechnol. Biofuels 8:82. doi: 10.1186/s13068-015-0265-4

Malasarn, D., Kropat, J., Hsieh, S. I., Finazzi, G., Casero, D., Loo, J. A.,et al. (2013). Zinc deficiency impacts CO2 assimilation and disrupts copper homeostasis in Chlamydomonas reinhardtii. J. Biol. Chem. 288, 10672-10683. doi: 10.1074/jbc.M113.455105

Mandalam, R. K., and Palsson, B. (1998). Elemental balancing of biomass and medium composition enhances growth capacity in highdensity Chlorella vulgaris cultures. Biotechnol. Bioeng. 59, 605-611. doi: 10.1002/(SICI) 1097-0290(19980905)59:5<605::AID-BIT11>3.0.CO;2-8

Martin, P., Dyhrman, S. T., Lomas, M. W., Poulton, N. J., and Van Mooy, B. A. S. (2014). Accumulation and enhanced cycling of polyphosphate by sargasso sea plankton in response to low phosphorus. Proc. Natl. Acad. Sci. 111, 8089-8094. doi: $10.1073 /$ pnas.1321719111
Martino, A. D., Meichenin, A., Shi, J., Pan, K., and Bowler, C. (2007). Genetic and phenotypic characterization of Phaeodactylum tricornutum (Bacillariophyceae) accessions. J. Phycol. 43, 992-1009. doi: 10.1111/j.1529-8817.2007.00384.x

Matsuda, Y., Nakajima, K., and Tachibana, M. (2011). Recent progresses on the genetic basis of the regulation of $\mathrm{CO} 2$ acquisition systems in response to co2 concentration. Photosynth. Res. 109, 191-203. doi: 10.1007/s11120-0119623-7

McCloskey, D., Palsson, B. O., and Feist, A. M. (2013). Basic and applied uses of genome-scale metabolic network reconstructions of Escherichia coli. Mol. Syst. Biol. 9:661. doi: 10.1038/msb.2013.18

Merchant, S., and Helmann, J. (2012). Elemental economy: microbial strategies for optimizing growth in the face of nutrient limitation. Adv. Microb. Physiol. 60, 91-210. doi: 10.1016/B978-0-12-398264-3.00002-4

Mishra, P., Lee, N.-R., Lakshmanan, M., Kim, M., Kim, B.-G., and Lee, D.-Y. (2018). Genome-scale model-driven strain design for dicarboxylic acid production in Yarrowia lipolytica. BMC Syst. Biol. 12:12. doi: 10.1186/s12918-018-0542-5

Mokashi, K., Shetty, V., George, S. A., and Sibi, G. (2016). Sodium bicarbonate as inorganic carbon source for higher biomass and lipid production integrated carbon capture in Chlorella vulgaris. Achiev. Life Sci. 10, 111-117. doi: 10.1016/j.als.2016.05.011

Moore, M., Mills, M., Arrigo, K., Berman-Frank, I., Bopp, L., Boyd, P., et al. (2013). Processes and patterns of oceanic nutrient limitation. Nat. Geosci. 6, 701-710. doi: $10.1038 /$ ngeo 1765

Morel, F., Milligan, A., and Saito, M. (2003). Marine bioinorganic chemistry: the role of trace metals in the oceanic cycles of major nutrients. Treat. Geochem. 6 , 113-143. doi: 10.1016/B0-08-043751-6/06108-9

Morel, F., Reinfelder, J., Roberts, S., Chamberlain, C., Lee, J., and Yee, D. (1994). Zinc and carbon co-limitation of marine phytoplankton. Nature 369, 740-742. doi: $10.1038 / 369740 \mathrm{a} 0$

Moustafa, A., Beszteri, B., Maier, U. G., Bowler, C., Valentin, K., and Bhattacharya, D. (2009). Genomic footprints of a cryptic plastid endosymbiosis in diatoms. Science 324, 1724-1726. doi: 10.1126/science.1172983

Needoba, J. A., and Harrison, P. J. (2004). Influence of low light and a light: Dark cycle on NO3- uptake, intracellular NO3-, and nitrogen isotope fractionation by marine phytoplankton1. J. Phycol. 40, 505-516. doi: 10.1111/j.1529-8817.2004.03171.x

Ong, W. K., Courtney, D. K., Pan, S., Andrade, R. B., Kiley, P. J., Pfleger, B. F., et al. (2020). Model-driven analysis of mutant fitness experiments improves genome-scale metabolic models of Zymomonas mobilis ZM4. PLoS Comput. Biol. 16:e1008137. doi: 10.1371/journal.pcbi.1008137

Pan, S., and Reed, J. L. (2018). Advances in gap-filling genome-scale metabolic models and model-driven experiments lead to novel metabolic discoveries. Curr. Opin. Biotechnol. 51, 103-108. doi: 10.1016/j.copbio.2017.12.012

Peers, G., and Price, N. (2006). Copper-containing plastocyanin used for electron transport by an oceanic diatom. Nature 441, 341-344. doi: 10.1038/nature04630

Poolman, M. G. (2006). ScrumPy: metabolic modelling with Python. IEE Proc. Syst. Biol. 153, 375-378. doi: 10.1049/ip-syb:20060010

Poolman, M. G., Kundu, S., Shaw, R., and Fell, D. A. (2013). Responses to light intensity in a genome-scale model of rice metabolism. Plant Physiol. 162, 1060-1072. doi: 10.1104/pp.113.216762

Poolman, M. G., Miguet, L., Sweetlove, L. J., and Fell, D. A. (2009). A genome-scale metabolic model of Arabidopsis and some of its properties. Plant Physiol. 151, 1570-1581. doi: 10.1104/pp.109.141267

Pudney, A., Gandini, C., Economou, C., Smith, R., Goddard, P., Napier, J., et al. (2019). Multifunctionalizing the marine diatom Phaeodactylum tricornutum for sustainable co-production of omega-3 long chain polyunsaturated fatty acids and recombinant phytase. Sci. Rep. 9:11444. doi: $10.1038 / s 41598-019-47875-1$

Rashid, N., Rehman, M. S. U., and Han, J.-I. (2015). Enhanced growth rate and lipid production of freshwater microalgae by adopting two-stage cultivation system under diverse light and nutrients conditions. Water Environ. J. 29, 533-540. doi: $10.1111 /$ wej. 12110

Reinfelder, J., Kraepiel, A., and Morel, F. (2000). Unicellular C4 photosynthesis in a marine diatom. Nature 407, 996-999. doi: 10.1038/35039612

Richmond, A. (2000). Microalgal biotechnology at the turn of the millennium: a personal view. J. Appl. Phycol. 12, 441-451. doi: 10.1023/A:100812313 1307 
Roberts, K., Granum, E., Leegood, R. C., and Raven, J. A. (2007). C3 and C4 pathways of photosynthetic carbon assimilation in marine diatoms are under genetic, not environmental, control. Plant Physiol. 145, 230-235. doi: 10.1104/pp.107.102616

Saavedra, R., Muñoz, R., Taboada, M. E., Vega, M., and Bolado, S. (2018). Comparative uptake study of arsenic, boron, copper, manganese and zinc from water by different green microalgae. Bioresour. Technol. 263, 49-57. doi: 10.1016/j.biortech.2018.04.101

Sage, R. F. (2004). The evolution of c4 photosynthesis. New Phytol. 161, 341-370. doi: 10.1111/j.1469-8137.2004.00974.x

Schurmann, P., and Jacquot, J.-P. (2000). Plant thioredoxin systems revisited. Annu. Rev. Plant Physiol. Plant Mol. Biol. 51, 371-400. doi: 10.1146/annurev.arplant.51.1.371

Sibley, M. H., and Anderson, L. E. (1989). Light/Dark modulation of enzyme activity in developing barley leaves. Plant Physiol. 91, 1620-1624. doi: 10.1104/pp.91.4.1620

Singh, D., Carlson, R., Fell, D., and Poolman, M. (2015). Modelling metabolism of the diatom Phaeodactylum tricornutum. Biochem. Soc. Trans. 43, 1182-1186. doi: 10.1042/BST20150152

Sivakaminathan, S., Hankamer, B., Wolf, J., and Yarnold, J. (2018). Highthroughput optimisation of light-driven microalgae biotechnologies. Sci. Rep. 8:11687. doi: 10.1038/s41598-018-29954-x

Smith, S. R., Abbriano, R. M., and Hildebrand, M. (2012). Comparative analysis of diatom genomes reveals substantial differences in the organization of carbon partitioning pathways. Algal Res. 1, 2-16. doi: 10.1016/j.algal.2012.04.003

Sunda, W. G., and Huntsman, S. A. (1996). Antagonisms between cadmium and zinc toxicity and manganese limitation in a coastal diatom. Limnol. Oceanogr. 41, 373-387. doi: 10.4319/lo.1996.41.3.0373

Takács, I., Vanrolleghem, P., Wett, B., and Murthy, S. (2007). Elemental balance based methodology to establish reaction stoichiometry in environmental modeling. Water Sci. Technol. 56, 37-41. doi: 10.2166/wst.2007.606

Tejera, N., Crossman, L., Pearson, B., Stoakes, E., Nasher, F., Djeghout, B., et al. (2020). Genome-scale metabolic model driven design of a defined medium for Campylobacter jejuni M1cam. Front. Microbiol. 11:1072. doi: 10.3389/fmicb.2020.01072

van der Ark, K. C. H., Aalvink, S., Suarez-Diez, M., Schaap, P. J., de Vos, W. M., and Belzer, C. (2018). Model-driven design of a minimal medium for Akkermansia muciniphila confirms mucus adaptation. Microb. Biotechnol. 11, 476-485. doi: 10.1111/1751-7915.13033

Vandystadt, G., Bujaldon, S., Wollman, F.-A., Dubois, R., Roussel, P., Alric, J., et al. (2009). A new setup for in vivo fluorescence imaging of photosynthetic activity. Photosynth. Res. 102, 85-93. doi: 10.1007/s11120-009-9487-2

Varma, A., and Palsson, B. O. (1994). Stoichiometric flux balance models quantitatively predict growth and metabolic by-product secretion in wild-type Escherichia coli W3110. Appl. Environ. Microbiol. 60, 3724-3731. doi: 10.1128/AEM.60.10.3724-3731.1994

Villanova, V., Fortunato, A. E., Singh, D., Bo, D. D., Conte, M., Obata, T., et al. (2017). Investigating mixotrophic metabolism in the model diatom Phaeodactylum tricornutum. Philos. Trans. R. Soc. B 372:20160404. doi: 10.1098/rstb.2016.0404

Wang, J., and Seibert, M. (2017). Prospects for commercial production of diatoms. Biotechnol. Biofuels 10:16. doi: 10.1186/s13068-017-0699-y

Wijffels, R. H., and Barbosa, M. J. (2010). An outlook on microalgal biofuels. Science 329, 796-799. doi: 10.1126/science.1189003

Wilhelm, C., Bchel, C., Fisahn, J., Goss, R., Jakob, T., Laroche, J., et al. (2006). The regulation of carbon and nutrient assimilation in diatoms is significantly different from green algae. Protist 157, 91-124. doi: $10.1016 /$ j.protis.2006.02.003

Yang, Y.-H., Du, L., Hosokawa, M., Miyashita, K., Kokubun, Y., Arai, H., et al. (2017). Fatty acid and lipid class composition of the microalga Phaeodactylum tricornutum. J. Oleo Sci. 66, 363-368. doi: 10.5650/jos.ess16205

Yi, Z., Xu, M., Di, X., Brynjolfsson, S., and Fu, W. (2017). Exploring valuable lipids in diatoms. Front. Mar. Sci. 4:17. doi: 10.3389/fmars.2017.00017

Yodsuwan, N., Sawayama, S., and Sirisansaneeyakul, S. (2017). Effect of nitrogen concentration on growth, lipid production and fatty acid profiles of the marine diatom Phaeodactylum tricornutum. Agric. Nat. Resour. 51, 190-197. doi: 10.1016/j.anres.2017.02.004

Zaslavskaia, L. A., Lippmeier, J. C., Shih, C., Ehrhardt, D., Grossman, A. R., and Apt, K. E. (2001). Trophic conversion of an obligate photoautotrophic organism through metabolic engineering. Science 292, 2073-2075. doi: $10.1126 /$ science. 160015

Zhu, L. (2015). Microalgal culture strategies for biofuel production: a review. Biofuels Bioprod. Biorefin. 9, 801-814. doi: 10.1002/bbb. 1576

Conflict of Interest: VV was employed by company Fermentalg SA.

The remaining authors declare that the research was conducted in the absence of any commercial or financial relationships that could be construed as a potential conflict of interest.

Copyright (c) 2021 Villanova, Singh, Pagliardini, Fell, Le Monnier, Finazzi and Poolman. This is an open-access article distributed under the terms of the Creative Commons Attribution License (CC BY). The use, distribution or reproduction in other forums is permitted, provided the original author(s) and the copyright owner(s) are credited and that the original publication in this journal is cited, in accordance with accepted academic practice. No use, distribution or reproduction is permitted which does not comply with these terms. 